\title{
QUANTUM STOCHASTIC COCYCLES AND COMPLETELY BOUNDED SEMIGROUPS ON OPERATOR SPACES
}

\author{
J. MARTIN LINDSAY AND STEPHEN J. WILLS
}

\begin{abstract}
An operator space analysis of quantum stochastic cocycles is undertaken. These are cocycles with respect to an ampliated CCR flow, adapted to the associated filtration of subspaces, or subalgebras. They form a noncommutative analogue of stochastic semigroups in the sense of Skorohod. One-to-one correspondences are established between classes of cocycle of interest and corresponding classes of one-parameter semigroups on associated matrix spaces. Each of these 'global' semigroups may be viewed as the expectation semigroup of an associated quantum stochastic cocycle on the corresponding matrix space. The classes of cocycle covered include completely positive contraction cocycles on an operator system, or $C^{*}$-algebra; completely contractive cocycles on an operator space; and contraction operator cocycles on a Hilbert space. As indicated by Accardi and Kozyrev, the Schur-action matrix semigroup viewpoint circumvents technical (domain) limitations inherent in the theory of quantum stochastic differential equations. An infinitesimal analysis of quantum stochastic cocycles from the present wider perspective is given in a sister paper.
\end{abstract}

\section{INTRODUCTION}

Cocycles arise in classical and quantum probability theory, for studying Markov processes and solutions of linear (quantum) stochastic differential equations ([Sko], [Pin], [L]), and in the study of $E_{0}$-semigroups and product systems, as a means of perturbing such semigroups $\left(\left[\mathrm{Ar}_{2}\right]\right)$. In the former context they are known classically as stochastic semigroups. The core algebraic notion is as follows. Let $\theta=\left(\theta_{t}\right)_{t \geqslant 0}$ be a semigroup of linear maps on a vector space $V$, thus $\theta_{0}=\mathrm{id}$ and $\theta_{s+t}=\theta_{s} \circ \theta_{t}$ for all $s, t \geqslant 0$. Then a family $J=\left(J_{t}\right)_{t \geqslant 0}$ of linear maps on $V$ is a $\theta$-cocycle if the composed maps $\left(J_{t} \circ \theta_{t}\right)_{t \geqslant 0}$ again form a one-parameter semigroup, that is

$$
J_{0}=\mathrm{id}, \quad J_{s+t} \circ \theta_{s+t}=J_{s} \circ \theta_{s} \circ J_{t} \circ \theta_{t} \quad \text { for all } s, t \geqslant 0 .
$$

Further structure is usually imposed, in particular $V$ is often taken to be an operator algebra $A$ and each $\theta_{t}$ a ${ }^{*}$-homomorphism. For example in the case of $E_{0}$-semigroups, $\mathrm{A}=B(\mathrm{~h})$ for an infinite-dimensional separable Hilbert space $\mathrm{h}$, each $\theta_{t}$ is unital and *-homomorphic, and $J_{t}$ is given by $J_{t}=\operatorname{ad} U_{t}$, where $U$ is a unitary operator (left) $\theta$-cocycle, that is a family of unitaries

2000 Mathematics Subject Classification. Primary 81S25, 46L07, 47D06.

Key words and phrases. Quantum stochastic cocycle, CCR flow, E-semigroup, operator space, operator system, matrix space, completely bounded, completely positive. 
$\left(U_{t}\right)_{t \geqslant 0}$ on $\mathrm{h}$ satisfying

$$
U_{0}=I_{\mathrm{h}}, \quad U_{s+t}=U_{s} \theta_{s}\left(U_{t}\right) \quad \text { for all } s, t \geqslant 0
$$

(which implies (0.1)). For application to models of quantum spin systems, $V$ might be a uniformly hyperfinite $C^{*}$-algebra with $J$ consisting of completely positive contractions $\left(\left[\mathrm{AK}_{1}\right]\right)$ or $*$-homomorphisms $([\mathrm{BeW}])$. For applications to noncommutative geometry, $V$ would naturally be the norm-closure of the smooth algebra of a spectral triple with $J$ consisting of *-homomorphisms describing a Brownian motion on the noncommutative manifold ([SiG]). In quantum optics many examples arise in which $V$ is of the form $B(\mathrm{~h})$ and $J$ is given by conjugation by a unitary operator cocycle (0.2) (see [BHS], and references therein).

Note that in (0.1) each $J_{t}$ need only be defined on the image of $\theta_{t}$. Moreover to incorporate concepts such as Markovianity into this picture $V$ must be equipped with a time localisation, or filtration, with respect to which the maps $\theta_{t}$ are adapted; this leads to further simplification in the description of the maps $J_{t}$ which we outline next, without yet being precise about the types of tensor products involved. Fix Hilbert spaces $\mathfrak{h}$ and k. For measurable subsets $I \subset J$ of $\mathbb{R}_{+}$and $t>0$, let $\mathcal{F}_{I}$ denote the symmetric Fock space over $L^{2}(I ; \mathrm{k})$, and set $\mathcal{F}:=\mathcal{F}_{\mathbb{R}_{+}}$. Then we have the decomposition and inclusion

$$
\mathfrak{h} \otimes \mathcal{F}=\mathfrak{h} \otimes \mathcal{F}_{[0, t]} \otimes \mathcal{F}_{[t, \infty[}, \quad \mathcal{F}_{I} \cong \mathcal{F}_{I} \otimes \varpi\left(0_{J \backslash I}\right) \subset \mathcal{F}_{J}
$$

where $\varpi\left(0_{J \backslash I}\right)$ is the vacuum vector $(1,0,0, \ldots)$ in $\mathcal{F}_{J \backslash I}$. The right shift by $t$ on $L^{2}\left(\mathbb{R}_{+} ; \mathrm{k}\right)$ induces a unitary operator $\mathfrak{h} \otimes \mathcal{F} \rightarrow \mathfrak{h} \otimes \mathcal{F}_{[t, \infty[}$ by second quantisation and ampliation, allowing the identification of each operator $A \in B(\mathfrak{h} \otimes \mathcal{F})$ with an operator on $\mathfrak{h} \otimes \mathcal{F}_{[t, \infty[}$, and thence an operator $\sigma_{t}(A)$ on $\mathfrak{h} \otimes \mathcal{F}$ after ampliating with the identity operator on $\mathcal{F}_{[0, t[}$. The resulting family $\sigma=\left(\sigma_{t}\right)_{t \geqslant 0}$ is an $E_{0}$-semigroup - the $B(\mathfrak{h})$-ampliated CCR flow of index $k$.

Suppose now that $\left(j_{t}\right)_{t \geqslant 0}$ is a family of linear maps from a subalgebra $\mathcal{A}$ of $B(\mathfrak{h})$ to $B(\mathfrak{h} \otimes \mathcal{F})$ satisfying the initial condition and adaptedness property

$$
j_{0}(a)=a \otimes I_{\mathcal{F}}, \quad j_{t}(\mathcal{A}) \subset \mathcal{A} \otimes B\left(\mathcal{F}_{[0, t]}\right) \subset \mathcal{A} \otimes B(\mathcal{F}), \quad \text { for all } t \geqslant 0 .
$$

Let $\widehat{\jmath}_{t}$ denote the tensor product of $j_{t}$ with the identity map of $B\left(\mathcal{F}_{[t, \infty[}\right)$, so that $\widehat{\jmath}_{t}: \mathcal{A} \otimes B\left(\mathcal{F}_{[t, \infty[}\right)=\sigma_{t}(\mathcal{A} \otimes B(\mathcal{F})) \rightarrow \mathcal{A} \otimes B\left(\mathcal{F}_{[0, t]}\right) \otimes B\left(\mathcal{F}_{[t, \infty[}\right)=$ $\mathcal{A} \otimes B(\mathcal{F})$. Then (formally, for now) we see that $\left(\widehat{\jmath}_{t}\right)_{t \geqslant 0}$ is a $\sigma$-cocycle if and only if

$$
j_{s+t}=\widehat{\jmath}_{s} \circ \sigma_{s} \circ j_{t} \quad \text { for all } s, t \geqslant 0 .
$$

The identity (0.3) was taken as the definition of Markovian cocycle in the Fock space setting in $[\mathrm{Bra}]$, and the subsequent papers $[\mathrm{AcM}],\left[\mathrm{LW}_{2}\right],\left[\mathrm{AK}_{2}\right]$. Such a family $\left(j_{t}\right)_{t \geqslant 0}$ is also a quantum stochastic process in the sense of [AFL], and the family $\left(\mathbb{E}_{0} \circ j_{t}\right)_{t \geqslant 0}$, where $\mathbb{E}_{0}$ is the map $A \mapsto(\operatorname{id} \otimes \omega)(A)$ for the vector state $\omega: T \mapsto\langle\varpi(0), T \varpi(0)\rangle$, forms a semigroup on $\mathcal{A}$, the (vacuum) expectation semigroup of $j$.

The purpose of the papers [Bra], $[\mathrm{AcM}]$ and $\left[\mathrm{LW}_{2}\right]$ is to show that Markovian cocycles can be constructed by solving quantum stochastic differential equations, and that, under certain regularity assumptions, all such cocycles arise in this manner. Of course, one must be precise about the nature of the tensor products and the definition of the extension $\widehat{\jmath}_{t}$. In $[\mathrm{Bra}]$ and $[\mathrm{AcM}]$ 
this is achieved by assuming that the algebra $\mathcal{A}$ is a von Neumann algebra and that the $j_{t}$ are unital, injective, normal ${ }^{*}$-homomorphisms. These ideas were then extended to the $C^{*}$-algebraic setting in $\left[\mathrm{LW}_{2}\right]$ in a way that allows considerable freedom for the kind of tensor product used, and permits the study of cocycles for which no assumption about the properties of each $j_{t}$ is made - indeed cocycles for which the families $\left(j_{t}(a)\right)_{t \geqslant 0}$ consist of unbounded operators naturally arise, and these could be handled.

The main distinction between $[\mathrm{Bra}]$ and $\left[\mathrm{LW}_{2}\right]$ on the one hand, and $[\mathrm{AcM}]$ on the other, is the choice of regularity condition on the expectation semigroup of the cocycle; in the first two it is assumed to be norm-continuous, which leads to a stochastic generator all of whose components (with respect to any basis for $\mathrm{k}$ ) are bounded operators on $\mathcal{A}$. In $[\mathrm{AcM}]$ the natural assumption of pointwise ultraweak continuity of the semigroup is made, along with the further assumption that the domain of the generator contains a *-subalgebra as a core. There are examples of interest for which the second assumption does not hold ([ $\left.\left.\mathrm{Ar}_{1}\right],[\mathrm{Fag}]\right)$.

For each cocycle $j$ there is a family of associated semigroups $\left\{\left(\mathcal{P}_{t}^{x, y}\right)_{t \geqslant 0}\right.$ : $x, y \in \mathrm{k}\}$ on $\mathcal{A}$, which may individually be viewed as expectation semigroups of perturbations of the cocycle. In turn, these determine the cocycle through its 'semigroup decomposition' ([LiP], $\left.\left[\mathrm{LW}_{2}\right]\right)$. By a theorem of Parthasarathy-Sunder and Skeide (see Section 3) the cocycle is actually determined by a small number of associated semigroups- $(1+d)^{2}$ in case $\operatorname{dim} \mathrm{k}=d$ (see Proposition 4.1). Working with completely positive unital cocycles on a full algebra $\mathcal{A}=B(\mathfrak{h})$ and with one dimension of noise $(\mathrm{k}=\mathbb{C})$, Accardi and Kozyrev exploit the fact that the four semigroups $\left\{\left(\mathcal{P}_{t}^{x, y}\right)_{t \geqslant 0}: x, y \in\{0,1\} \subset \mathbb{C}\right\}$ determine a single semigroup acting componentwise on $\mathrm{M}_{2}(\mathcal{A})$, with Schur-action:

$$
\mathcal{P}_{t}^{\eta}:\left[a_{x y}\right] \mapsto\left[\mathcal{P}_{t}^{x, y}\left(a_{x y}\right)\right],
$$

which is completely positive and satisfies a certain normalisation condition arising from unitality of the cocycle $\left(\left[\mathrm{AK}_{2}\right]\right)$. More significantly they show that, from any such semigroup on $\mathrm{M}_{2}(\mathcal{A})$, a completely positive unital cocycle on $B(\mathcal{A})$ may be constructed whose associated semigroups include the component semigroups $\left(\mathcal{P}^{x, y}\right)$. This provides a new method for constructing cocycles circumventing problems with the direct approach via quantum stochastic calculus which is hampered by inherent domain constraints on the coefficients of the quantum stochastic differential equation when these coefficients are unbounded. Indeed, in the only previous result in this direction $([\mathrm{FaS}])$, this is met by the severe assumption that a common domain is left invariant by all coefficients of the QSDE. The significant advance here is that there is no longer need for the domains of the components of the stochastic generators to directly match up, let alone to intersect in a common core. In fact, as we make clear in $\left[\mathrm{LW}_{5}\right]$, for the infinitesimal analysis of QS cocycles, attention may fruitfully be shifted to the generator of the global semigroup (0.4). Potential applications of this viewpoint in the theory of classical SDEs are explored in [AFR].

In this paper we use ideas from the theory of operator spaces to make a thorough analysis of quantum stochastic cocycles, establishing and exploiting correspondences between classes of cocycle of interest and corresponding 
classes of global semigroups on associated matrix spaces (see Section 2). Matrix spaces are a hybrid form of operator space Fubini product in the sense of Tomiyama ([Tom], $[\mathrm{EfR}])$ which splice together norm and ultraweak topologies $\left(\left[\mathrm{LW}_{3}\right],[\mathrm{LiT}]\right)$.

For an operator space $\mathrm{V}$ in $B\left(\mathfrak{h}_{1} ; \mathfrak{h}_{2}\right)$, we analyse $(0.3)$, for a family of linear maps $\left(j_{t}: \mathrm{V} \rightarrow B\left(\mathfrak{h}_{1} \otimes \mathcal{F} ; \mathfrak{h}_{2} \otimes \mathcal{F}\right)\right)_{t \geqslant 0}$ on which our only assumptions are that they be completely bounded and map into the $\mathcal{F}$-matrix space over $\mathrm{V}$. Both assumptions are natural; the first covers all cases of interest, including that of QS flows on an operator algebra, and QS contraction cocycles on a Hilbert space; the second is a precise formulation of the cocycle being "on V". This matrix space viewpoint, achieved by use of a hybrid topology created through allowing differing topologies on each tensor factor, permits a unified treatment of cocycles on a wide variety of structures, all the time respecting the measure-theoretic nature of the noise. Cases covered include cocycles on operator spaces, $C^{*} / W^{*}$-algebras, operator systems, Hilbert $C^{*}$ modules, coalgebras, quantum (semi)groups and Hilbert spaces. The latter class, of bounded operator cocycles, consists of adapted families $X=\left(X_{t}\right)_{t \geqslant 0}$ in $B(\mathfrak{h} \otimes \mathcal{F})$ satisfying $(0.2)$ for $\theta=\sigma$; they are in one-to-one correspondence with the completely bounded cocycles on the column operator space $B(\mathbb{C} ; \mathfrak{h})$.

Matrix spaces also enable the study of cocycles with arbitrary noise dimension space k. The global semigroup $(0.4)$ is then defined on an $l^{2}(\mathrm{~T})$ matrix space over $\mathrm{V}$, where $\mathrm{T}$ might be an orthonormal basis for $\mathrm{k}$ augmented by the zero of $k$. These semigroups are incorporated into our approach to quantum stochastics $\left(\left[\mathrm{LW}_{2-4}\right]\right)$ by being realised as vacuum expectation semigroups of cocycles (on the corresponding matrix space) obtained by perturbing matrix-space liftings of the original cocycle (Proposition 4.9). Crucially this process maintains complete positivity when the cocycle is on an operator system or $C^{*}$-algebra, and encodes contractivity and unitality in recoverable ways. Accardi and Kozyrev have outlined an extension of their theory to multidimensional noise in $\left[\mathrm{AK}_{3}\right]$.

In the last section (Section 5) we prove a number of characterisation and reconstruction results extending the one given in $\left[\mathrm{AK}_{2}\right]$. We provide results for the following cases: completely positive contraction cocycles on operator systems and $C^{*}$-algebras; completely positive unital cocycles on operator systems; completely contractive cocycles on operator spaces; contraction operator cocycles, and positive contraction operator cocycles, on Hilbert spaces. Liebscher has obtained an alternative characterisation of uniformly bounded operator quantum stochastic cocycles ([Lie]).

In this paper we have focused on structural questions for cocycles. In a sister paper $\left(\left[\mathrm{LW}_{5}\right]\right)$ we analyse the generation of quantum stochastic cocycles from the perspective of their global semigroups, and relate these to characterisations by means of quantum stochastic differential equations. In particular all issues pertaining to continuity of the map $t \mapsto j_{t}$ are deferred to that paper. The global semigroup viewpoint developed here has already been used to obtain new results on (operator) quantum stochastic differential equations $\left(\left[\mathrm{LW}_{4}\right]\right)$. However, the essential point, as emphasised by Accardi and Kozyrev, is that this viewpoint allows one to overcome inherent limitations of quantum stochastic calculus. This has been carried out for 
holomorphic contraction operator cocycles in [LiS]. In that paper an infinitesimal characterisation of these cocycles is given, which manifestly exceeds the scope of quantum stochastic differential equations.

Finally we mention that families of Schur-action semigroups of completely positive maps of the type arising in this paper also appear in the context of product systems of Hilbert modules where they play a natural role in the analysis of such systems of "Type I" ([BBLS]).

General notational conventions. Given a vector-valued function $f: S \rightarrow V$ defined on a set $S$, and subset $A$ of $S$, we write $f_{A}$ for the function $\mathbf{1}_{A} f$ : $S \rightarrow V$ where $\mathbf{1}_{A}$ denotes the indicator function of $A$. All linear spaces are complex, and Hilbert space inner products are linear in their second argument and thereby consistent with the standard convention for Hilbert $C^{*}$-modules. The $n$-fold orthogonal sum of a Hilbert space $\mathrm{h}$ is denoted $\mathrm{h}^{n}$. We use the notation $B(X ; Y)$ for the normed space of bounded operators between normed spaces $X$ and $Y$. For an index set $I$ and a vector space $V, \mathrm{M}_{I}(V)$ denotes the linear space of matrices $\left[a_{j}^{i}\right]_{i, j \in I}$ with entries from $V$, and $\mathrm{M}_{n}(V)$ the linear space of $n \times n$ matrices with entries from $V$, so that $\mathrm{M}_{n}(V) \cong \mathrm{M}_{\{1, \ldots, n\}}(V) \cong V \underline{\otimes} \mathrm{M}_{n}(\mathbb{C})$, where $\underline{\otimes}$ denotes the linear tensor product. For a map $\phi: V \rightarrow W$ its matrix lifting $\mathrm{M}_{n}(V) \rightarrow \mathrm{M}_{n}(W)$, $\left[a_{j}^{i}\right] \mapsto\left[\phi\left(a_{j}^{i}\right)\right]$ is denoted $\phi^{(n)}$. The symbol $\otimes$ is used for the tensor product of vectors, Hilbert spaces and Hilbert space operators; we also use it for spatial/injective tensor products of operator spaces and completely bounded maps; $\bar{\otimes}$ is used for ultraweak tensor products of ultraweakly closed operator spaces and ultraweakly continuous completely bounded maps between such spaces. Finally $\delta$ is reserved for the Kronecker symbol, thus $\left(\delta^{i}\right)_{i \in I}$ denotes the standard orthonormal basis of $l^{2}(I)$.

\section{Operator spaces}

In this section we establish some notation and terminology, introduce some useful topologies and recall some results from operator space theory ([BLM], [EfR], [Pau], [Pis]). We freely use the abbreviations CB, CP, CC and CI for completely bounded, completely positive, completely contractive and completely isometric, and $\mathrm{CPC}$ for completely positive and contractive.

Dirac and $E$-notations. For a Hilbert space h, we denote $B(\mathbb{C} ; \mathrm{h})$ and $B(\mathrm{~h} ; \mathbb{C})$ by $|\mathrm{h}\rangle$ and $\langle\mathrm{h}|$ respectively, and for $e \in \mathrm{h}$ we write $|e\rangle \in|\mathrm{h}\rangle$ and $\langle e| \in\langle\mathrm{h}|$ for the operators defined by

$$
|e\rangle \lambda=\lambda e \text { and }\langle e| f=\langle e, f\rangle .
$$

Thus $|d\rangle\langle e|$ is the rank one operator in $B(\mathrm{~h})$ given by $f \mapsto\langle e, f\rangle d$. We usually abbreviate $I_{\mathrm{H}} \otimes|e\rangle$ and $|e\rangle \otimes I_{\mathrm{H}}$ to $E_{e}$, and write $E^{e}$ for $\left(E_{e}\right)^{*}$, allowing context to reveal both the Hilbert space $\mathrm{H}$ and the order of the tensor components.

Matrices of operators. For Hilbert spaces $\mathrm{H}, \mathrm{K}$ and $\mathrm{h}$, a choice of orthonormal basis $\kappa=\left(e_{i}\right)_{i \in I}$ for $\mathrm{h}$ entails isometric isomorphisms $\mathrm{H} \otimes \mathrm{h} \rightarrow$ 
$\bigoplus_{i \in I} \mathrm{H}$ and $\mathrm{K} \otimes \mathrm{h} \rightarrow \bigoplus_{i \in I} \mathrm{~K}$ given by $\xi \mapsto{ }^{\kappa} \xi:=\left(E^{(i)} \xi\right)_{i \in I}$, and a linear injection

$$
B(\mathrm{H} \otimes \mathrm{h} ; \mathrm{K} \otimes \mathrm{h}) \rightarrow \mathrm{M}_{I}(B(\mathrm{H} ; \mathrm{K})), \quad T \mapsto{ }^{\kappa} T:=\left[E^{(i)} T E_{(j)}\right]_{i, j \in I}
$$

where $E_{(j)}:=I_{\mathrm{H}} \otimes\left|e_{j}\right\rangle: \mathrm{H} \rightarrow \mathrm{H} \otimes \mathrm{h}$ and $E^{(i)}:=\left(E_{(i)}\right)^{*}: \mathrm{K} \otimes \mathrm{h} \rightarrow \mathrm{K}$. We denote by $\mathrm{M}_{I}(B(\mathrm{H} ; \mathrm{K}))_{\mathrm{b}}$ the image of the map (1.1); the resulting matrices act as operators from $\bigoplus_{i \in I} \mathrm{H}=\mathrm{H} \otimes l^{2}(I)$ to $\bigoplus_{i \in I} \mathrm{~K}=\mathrm{K} \otimes l^{2}(I)$ as matrices should: if $A=\left[a_{j}^{i}\right]_{i, j \in I}$ then

$$
A v=\left(\sum_{j \in I} a_{j}^{i} v^{j}\right)_{i \in I} .
$$

Thus the subspace $\mathrm{M}_{I}(B(\mathrm{H} ; \mathrm{K}))_{\mathrm{b}}$ is identified with $B\left(\bigoplus_{i \in I} \mathrm{H} ; \bigoplus_{i \in I} \mathrm{~K}\right)=$ $B\left(\mathrm{H} \otimes l^{2}(I) ; \mathrm{K} \otimes l^{2}(I)\right)$, cf. the familiar identification

$$
\mathrm{M}_{n}(B(\mathrm{H} ; \mathrm{K}))=B\left(\mathrm{H}^{n} ; \mathrm{K}^{n}\right)=B\left(\mathrm{H} \otimes \mathbb{C}^{n} ; \mathrm{K} \otimes \mathbb{C}^{n}\right) .
$$

The following characterisation of nonnegative operators on an orthogonal direct sum will be useful ([FoF], [GLSW]):

$$
\begin{array}{r}
B(\mathrm{H} \oplus \mathrm{K})_{+}=\left\{\left[\begin{array}{cc}
A & B \\
B^{*} & D
\end{array}\right]: A \in B(\mathrm{H})_{+}, D \in B(\mathrm{~K})_{+} \text {and } B=A^{1 / 2} R D^{1 / 2}\right. \\
\text { for some contraction } R \in B(\mathrm{~K} ; \mathrm{H})\} . \quad(1.3)
\end{array}
$$

It follows easily that if $A=\left[A_{i j}\right] \in B\left(\mathrm{H}^{n}\right)=\mathrm{M}_{n}(B(\mathrm{H}))$ is nonnegative with zero diagonal entries then $A=0$.

h-matrix topologies. The study of quantum stochastic cocycles on $C^{*}$ algebras raises the question of how the topology of the algebra should be spliced with the measure theoretic noise. This leads naturally to the consideration of two hybrid topologies which we describe next. The h-ultraweak (respectively, h-weak operator) topology on $B(\mathrm{H} \otimes \mathrm{h} ; \mathrm{K} \otimes \mathrm{h}$ ) is the locally convex topology defined by the seminorms

$$
p^{\omega}: T \mapsto\|\Omega(T)\|, \omega \in B(\mathrm{~h})_{*} \quad\left(\text { resp. } p_{e, d}: T \mapsto\left\|E^{e} T E_{d}\right\|, d, e \in \mathrm{h}\right),
$$

where $\Omega$ is the slice map

$$
\operatorname{id}_{B(\mathrm{H} ; \mathrm{K})} \bar{\otimes} \omega: B(\mathrm{H} \otimes \mathrm{h} ; \mathrm{K} \otimes \mathrm{h}) \rightarrow B(\mathrm{H} ; \mathrm{K})
$$

$([\mathrm{KaR}])$. Note that $p_{e, d}=p^{\omega}$ for the vector functional $\omega=\omega_{e, d}: T \mapsto$ $\langle e, T d\rangle$. Thus, in the partial ordering of topologies on $B(\mathrm{H} \otimes \mathrm{h} ; \mathrm{K} \otimes \mathrm{h})$,

h-W.O.T. $\leqslant$ h-ultraweak topology $\leqslant$ norm topology.

The h-ultraweak (respectively h-weak operator) topology coincides with the ultraweak (resp. weak operator) topology if $B(\mathrm{H} ; \mathrm{K})$ is finite dimensional, and coincides with the norm topology if $h$ is finite dimensional. On bounded sets the h-ultraweak and $\mathrm{h}$-w.o. topologies coincide and are, in general, finer than the ultraweak topology. If $\mathrm{V}$ and $\mathrm{W}$ are subspaces of $B(\mathrm{H} ; \mathrm{K})$ and $B(\mathrm{~h})$ respectively, with $\mathrm{V}$ norm closed, then

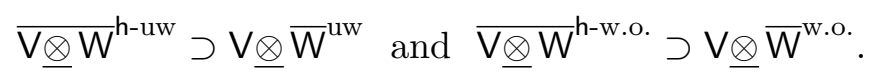

Since for each $T \in B(\mathrm{H} \otimes \mathrm{h} ; \mathrm{K} \otimes \mathrm{h})$ the bounded net

$$
\left\{\left(I_{\mathrm{K}} \otimes P_{F}\right) T\left(I_{\mathbf{H}} \otimes P_{F}\right): F \text { subspace of } \mathrm{h}, \operatorname{dim} F<\infty\right\}
$$


converges h-weakly to $T, B(\mathrm{H} ; \mathrm{K}) \otimes B(\mathrm{~h})$ is h-ultraweakly dense in $B(\mathrm{H} \otimes$ $h ; K \otimes h)$.

Operator spaces. In this paper operator spaces will take concrete form, as closed subspaces of the space of bounded operators between two Hilbert spaces, with one class of exceptions. We refer to $\mathrm{V}$ being an operator space in $B(\mathrm{~h} ; \mathrm{k}) ; \mathrm{M}_{n}(\mathrm{~V})$ is thus normed by being identified with a closed subspace of $B\left(\mathrm{~h}^{n} ; \mathrm{k}^{n}\right)$ through the identifications (1.2). The exception is that, for concrete operator spaces $\mathrm{V}$ and $\mathrm{W}$, the Banach space of completely bounded operators $C B(\mathrm{~V} ; \mathrm{W})$ is endowed with matrix norms making it an operator space via the linear isomorphisms

$$
\mathrm{M}_{n}(C B(\mathrm{~V} ; \mathrm{W}))=C B\left(V ; \mathrm{M}_{n}(\mathrm{~W})\right)
$$

An operator space $\mathrm{V}$ in $B(\mathrm{~h} ; \mathrm{k})$ has a concrete adjoint operator space in $B(\mathrm{k} ; \mathrm{h})$ :

$$
\mathrm{V}^{\dagger}:=\left\{T^{*}: T \in \mathrm{V}\right\}, \quad T \mapsto T^{*} \text { (Hilbert space operator adjoint). }
$$

For concrete operator spaces $\mathrm{V}$ and $\mathrm{W}$ and map $\phi: \mathrm{V} \rightarrow \mathrm{W}$,

$$
\phi^{\dagger}: \mathrm{V}^{\dagger} \rightarrow \mathrm{W}^{\dagger}, \quad T^{*} \mapsto \phi(T)^{*},
$$

defines a map which is a completely bounded, respectively completely isometric, operator if $\phi$ is, moreover $\left(C B\left(\mathrm{~V}^{\dagger} ; \mathrm{W}^{\dagger}\right), \phi \mapsto \phi^{\dagger}\right)$ is the adjoint operator space of $C B(\mathrm{~V} ; \mathrm{W})$ (see $[\mathrm{BLM}])$. When $\mathrm{V}^{\dagger}=\mathrm{V}$ and $\mathrm{W}^{\dagger}=\mathrm{W}$, we call a map $\phi: \mathrm{V} \rightarrow \mathrm{W}$ real if it is adjoint-preserving, that is if it satisfies $\phi^{\dagger}=\phi$.

Operator systems. Recall that a (concrete) operator system is a closed subspace $\mathrm{V}$ of $B(\mathrm{~h})$, for some Hilbert space $\mathrm{h}$, which is closed under taking adjoints and contains $I_{\mathrm{h}}$. Thus an operator system is linearly generated by its nonnegative elements, and each $\mathrm{M}_{n}(\mathrm{~V})$ is itself an operator system in $B\left(\mathrm{~h}^{n}\right)$. Operator systems also have an abstract characterisation involving the order structure on the sequence $\left\{\mathrm{M}_{n}(\mathrm{~V}): n \geqslant 1\right\}$, due to Choi and Effros ([SiS], Theorem 1.2.7).

The following summarises and extends parts of Propositions 2.1 and 3.6 of $[\mathrm{Pau}]$.

Proposition 1.1. Let $\phi: \mathrm{V} \rightarrow \mathrm{W}$ be a positive linear map between operator systems. Then $\phi$ is bounded, real and satisfies $\|\phi\| \leqslant 2\|\phi(I)\|$. Moreover if $\phi$ is 2-positive then $\|\phi\|=\|\phi(I)\|$, and if $\phi$ is completely positive then it is completely bounded with $\|\phi\|_{\mathrm{cb}}=\|\phi(I)\|$.

Remark. In contrast to the situation with $C^{*}$-algebras, an example of Arveson ([Pau], Example 2.2) shows that for operator systems the factor of 2 cannot be removed. Note that a completely positive contraction is completely contractive.

The following construction, known as Paulsen's $2 \times 2$ matrix trick, provides a route by which operator system results can be applied to operator spaces. To each operator space $\mathrm{V}$ in $B\left(\mathrm{~h} ; \mathrm{h}^{\prime}\right)$ is associated the operator system

$$
\widetilde{\mathrm{V}}=\left\{\left[\begin{array}{cc}
\alpha I_{\mathrm{h}^{\prime}} & a \\
b & \beta I_{\mathrm{h}}
\end{array}\right]: \alpha, \beta \in \mathbb{C}, a \in \mathrm{V}, b \in \mathrm{V}^{\dagger}\right\}
$$


in $B\left(\mathrm{~h}^{\prime} \oplus \mathrm{h}\right)$, and each linear map $\phi: \mathrm{V} \rightarrow \mathrm{W}$, into an operator space in $B\left(\mathrm{~K} ; \mathrm{K}^{\prime}\right)$, gives rise to a linear, real, unital map

$$
\widetilde{\phi}: \widetilde{V} \rightarrow \widetilde{W}, \quad\left[\begin{array}{cc}
\alpha I_{\mathrm{h}^{\prime}} & a \\
b & \beta I_{\mathrm{h}}
\end{array}\right] \mapsto\left[\begin{array}{cc}
\alpha I_{\mathrm{K}^{\prime}} & \phi(a) \\
\phi^{\dagger}(b) & \beta I_{\mathrm{K}}
\end{array}\right]
$$

Corollary $1.2([\mathrm{Pau}]$, Lemma 8.1). Let $\phi: \mathrm{V} \rightarrow \mathrm{W}$ be a linear map between operator spaces. Then $\phi$ is completely contractive if and only if $\widetilde{\phi}$ is completely positive.

Remark. Since $\widetilde{\phi}$ is unital by construction, Proposition 1.1 implies that it is necessarily completely contractive if it is $\mathrm{CP}$.

The proof is instructive; it starts by noting that the equivalence of contractivity of $\phi$ and positivity of $\widetilde{\phi}$ follows from the characterisation (1.3); it proceeds by identifying $\left(\mathrm{h}^{\prime} \oplus \mathrm{h}\right)^{n}$ with $\left(\mathrm{h}^{\prime}\right)^{n} \oplus \mathrm{h}^{n}$, so that $\mathrm{M}_{n}(\widetilde{\mathrm{V}})$ consists of elements

$$
\left[\begin{array}{cc}
I_{\mathrm{h}^{\prime}} \otimes \lambda & A \\
B & I_{\mathrm{h}} \otimes \mu
\end{array}\right]
$$

in which $\lambda, \mu \in \mathrm{M}_{n}(\mathbb{C}), A \in \mathrm{M}_{n}(\mathrm{~V})$ and $B \in \mathrm{M}_{n}\left(\mathrm{~V}^{\dagger}\right)=\mathrm{M}_{n}(\mathrm{~V})^{\dagger}$; and concludes by noting that $\widetilde{\phi}^{(n)}$ corresponds to the extension of $\widetilde{\phi^{(n)}}$ which takes (1.6) to

$$
\left[\begin{array}{cc}
I_{\mathrm{K}^{\prime}} \otimes \lambda & \phi^{(n)}(A) \\
\phi^{(n) \dagger}(B) & I_{\mathrm{K}} \otimes \mu
\end{array}\right]
$$

and that this map is positive if $\widetilde{\phi^{(n)}}$ is.

Nonunital $C^{*}$-algebras. The prototypical example of an operator system is a unital $C^{*}$-algebra, acting nondegenerately on a Hilbert space h. Unfortunately the operator system axioms/definition exclude nonunital $C^{*}$-algebras (for extensions of the Choi-Effros characterisation in this direction see [Sch]). The following result, which is standard (e.g. [SiS], Theorem 1.2.1), is collected here for convenience.

Proposition 1.3. Let $\mathrm{C}$ be a nonunital $C^{*}$-algebra acting nondegenerately on a Hilbert space $\mathrm{h}$, and let 1 denote the identity of $B(\mathrm{~h})$, then the unital $C^{*}$-algebra

$$
{ }^{u} \mathrm{C}:=C^{*}(\mathrm{C} \cup\{1\})=\{a+z 1: a \in \mathrm{C}, z \in \mathbb{C}\}
$$

contains $\mathrm{C}$ as a maximal ideal. If $\phi: \mathrm{C} \rightarrow B(\mathrm{~K})$ is a linear completely positive map, for some Hilbert space $\mathrm{K}$, then $\|\phi\|=\sup \left\{\|\phi(a)\|: a \in \mathrm{C}_{+, 1}\right\}$ and for any $C \geqslant\|\phi\|$,

$$
\psi:{ }^{u} \mathrm{C} \rightarrow B(\mathrm{~K}), \quad a+z 1 \mapsto \phi(a)+z C I_{\mathrm{K}}
$$

defines a $C P$ extension of $\phi$ satisfying $\|\psi\|=C$.

From operators to CB maps. The next result provides the basic mechanism whereby operator cocycles will be viewed as completely bounded mapping cocycles.

Proposition 1.4. For Hilbert spaces $\mathrm{h}, \mathrm{k}, \mathrm{H}$ and $\mathrm{K}$, the identity

$$
\phi(|u\rangle)=X\left(|u\rangle \otimes I_{\mathrm{H}}\right) \quad u \in \mathrm{h},
$$


establishes a completely isometric isomorphism between the spaces $B(\mathrm{~h} \otimes$ $\mathrm{H} ; \mathrm{K})$ and $C B(|\mathrm{~h}\rangle ; B(\mathrm{H} ; \mathrm{K}))$. Similarly, $\psi(\langle u|)=\left(\langle u| \otimes I_{\mathrm{H}}\right) Y$ establishes a completely isometric isomorphism between $B(\mathrm{H} ; \mathrm{k} \otimes \mathrm{K})$ and $C B(\langle\mathrm{k}| ; B(\mathrm{H} ; \mathrm{K}))$.

Proof. Set $\mathrm{V}=B(\mathrm{~h} \otimes \mathrm{H} ; \mathrm{K})$ and $\mathrm{W}=C B(|\mathrm{~h}\rangle ; B(\mathrm{H} ; \mathrm{K}))$.

Let $\mathcal{T}:|\mathrm{h}\rangle \rightarrow B(\mathrm{H} ; \mathrm{h} \otimes \mathrm{H})$ denote the ampliation $|u\rangle \mapsto|u\rangle \otimes I_{\mathrm{H}}$, and for $X \in \mathrm{V}$ let $L_{X}$ denote the corresponding left multiplication operator $B(\mathrm{H} ; \mathrm{h} \otimes \mathrm{H}) \rightarrow B(\mathrm{H} ; \mathrm{K})$. Then $\mathcal{T}$ is CI, and $L_{X}$ is CB with $\left\|L_{X}\right\|_{\mathrm{cb}}=\|X\|$. Thus $X \mapsto \phi_{X}:=L_{X} \circ \mathcal{T}$ defines a contraction $\Phi=\Phi_{\mathrm{h}, \mathrm{H} ; \mathrm{K}}: \mathrm{V} \rightarrow \mathrm{W}$, with $\phi_{X}$ satisfying (1.7).

For $\phi \in \mathrm{W}$ let $X_{\phi}^{0}: \mathrm{h} \underline{\otimes} \mathrm{H} \rightarrow \mathrm{K}$ be the linearisation of the bilinear map $(u, f) \mapsto \phi(|u\rangle) f$. Then, for $\xi \in \mathbf{h} \otimes \mathbf{H}$, expressing $\xi$ in the form $\sum_{i=1}^{n} u_{i} \otimes f^{i}$ where $u_{1}, \ldots, u_{n}$ are mutually orthogonal unit vectors in $\mathrm{h}$,

$$
\left\|X_{\phi}^{0} \xi\right\|=\left\|\phi^{(n)}(T) \mathbf{f}\right\| \text { where } T=\left[\begin{array}{ccc}
\left|u_{1}\right\rangle & \cdots & \left|u_{n}\right\rangle \\
0 & \cdots & 0 \\
\vdots & \ddots & \\
0 & & 0
\end{array}\right] \text { and } \mathbf{f}=\left(\begin{array}{c}
f^{1} \\
\vdots \\
f^{n}
\end{array}\right) .
$$

Since $T \in B\left(\mathbb{C}^{n} ; \mathrm{h}^{n}\right)$ has norm one and $\|\mathbf{f}\|=\|\xi\|, X_{\phi}^{0}$ is bounded with norm at most $\|\phi\|_{\mathrm{cb}}$; let $X_{\phi} \in B(\mathrm{~h} \otimes \mathrm{H} ; \mathrm{K})$ be its continuous extension. Then $X_{\phi}$ satisfies (1.7) and $\phi \mapsto X_{\phi}$ defines a contraction $\Psi: \mathrm{W} \rightarrow \mathrm{V}$.

Clearly $\Phi$ and $\Psi$ are mutually inverse, thus they are isometric too, and so are Banach space isometric isomorphisms.

Now let $X=\left[X_{j}^{i}\right] \in \mathrm{M}_{n}(B(\mathrm{~h} \otimes \mathrm{H} ; \mathrm{K}))=B\left(\mathrm{~h} \otimes \mathrm{H}^{n} ; \mathrm{K}^{n}\right)$. Then, under the identification $\mathrm{M}_{n}(C B(|\mathrm{~h}\rangle ; B(\mathrm{H} ; \mathrm{K})))=C B\left(|\mathrm{~h}\rangle ; B\left(\mathrm{H}^{n} ; \mathrm{K}^{n}\right)\right)($ see (1.4))

$$
\left[X_{j}^{i}\left(|u\rangle \otimes I_{\mathrm{H}}\right)\right]=X\left(|u\rangle \otimes I_{\mathrm{H}^{n}}\right)=\phi_{X}(|u\rangle),
$$

and so $\left(\Phi_{\mathrm{h}, \mathrm{H} ; \mathrm{K}}\right)^{(n)}=\Phi_{\mathrm{h}, \mathrm{H}^{n} ; \mathrm{K}^{n}}$. It follows that $\Phi$ is a CI isomorphism.

The second isomorphism is implemented by $\left(\Phi_{\mathrm{k}, \mathrm{K} ; \mathrm{H}}\right)^{\dagger}$.

The next result details some traffic in the above correspondence.

Corollary 1.5. For Hilbert spaces $\mathrm{h}, \mathrm{H}$ and $\mathrm{K}$, let $X \in B(\mathrm{~h} \otimes \mathrm{H} ; \mathrm{K})$ and $\phi \in C B(|\mathrm{~h}\rangle ; B(\mathrm{H} ; \mathrm{K}))$ correspond according to (1.7).

(a) If $X$ is injective then $\phi$ is injective.

(b) If $X$ is isometric then $\phi$ is completely isometric.

The converses hold when $\mathrm{H}=\mathbb{C}$.

Proof. Identifying $\mathrm{L}^{n}$ with $\mathbb{C}^{n} \otimes \mathrm{L}$ for the Hilbert spaces $\mathrm{L}=\mathrm{H}, \mathrm{K}$, (a) and (b) follow from the identity

$$
\phi^{(n)}(T)=\left(I_{\mathbb{C}^{n}} \otimes X\right)\left(T \otimes I_{\mathrm{H}}\right), \quad T \in \mathrm{M}_{n}(|\mathrm{~h}\rangle), n \in \mathbb{N} .
$$

When $\mathrm{H}=\mathbb{C}, B(\mathrm{~h} ; \mathrm{K}) \cong C B(|\mathrm{~h}\rangle ;|\mathrm{K}\rangle)$, with $\phi(|u\rangle)=|X u\rangle$, from which the converses follow immediately.

Example. Let $\mathrm{K}=\mathrm{h} \otimes \mathrm{H}^{\prime}$ and $X=I_{\mathrm{h}} \otimes R$, where $R \in B\left(\mathrm{H} ; \mathrm{H}^{\prime}\right)$ is noninjective and of norm one, for some Hilbert space $\mathrm{H}^{\prime}$. Then $X$ is noninjective but, for each $n \in \mathbb{N}$,

$$
\phi_{X}^{(n)}(T)=T \otimes R, \quad T \in \mathrm{M}_{n}(|\mathrm{~h}\rangle),
$$

so $\phi_{X}$ is completely isometric. 


\section{Matrix SPACES}

In this section we describe an abstract matrix construction over a (concrete) operator space, with Hilbert space as index set, that was introduced in $\left[\mathrm{LW}_{3}\right]$, and we develop some of its properties. The matrix spaces considered here are a coordinate-free version of the spaces of infinite matrices over an operator space introduced by Effros and Ruan (see [EfR], Chapter 10). Maps between spaces of matrices that are comprised of a matrix of linear maps, each one mapping between corresponding components of the matrices (i.e. having Schur action), are then characterised.

Lemma 2.1. For an operator space $\mathrm{V}$ in $B(\mathrm{H} ; \mathrm{K})$ and total subsets $\mathrm{T}$ and $\mathrm{T}^{\prime}$ of a Hilbert space $\mathrm{h}$, we have

$$
\begin{aligned}
& \left\{T \in B(\mathrm{H} \otimes \mathrm{h} ; \mathrm{K} \otimes \mathrm{h}): E^{x} T E_{y} \in \mathrm{V} \text { for all } x \in \mathrm{T}^{\prime}, y \in \mathrm{T}\right\} \\
= & \left\{T \in B(\mathrm{H} \otimes \mathrm{h} ; \mathrm{K} \otimes \mathrm{h}):\left(\mathrm{id}_{B(\mathrm{H} ; \mathrm{K})} \bar{\otimes} \omega\right)(T) \in \mathrm{V} \text { for all } \omega \in B(\mathrm{~h})_{*}\right\} .
\end{aligned}
$$

Proof. This follows from the following facts. For $T \in B(\mathrm{H} \otimes \mathrm{h} ; \mathrm{K} \otimes \mathrm{h})$, $E^{x} T E_{y}=\left(\operatorname{id}_{B(\mathrm{H} ; \mathrm{K})} \bar{\otimes} \omega_{x, y}\right)(T)$, the set $\left\{\omega \in B(\mathrm{~h})_{*}:\left(\mathrm{id}_{B(\mathrm{H} ; \mathrm{K})} \bar{\otimes} \omega\right)(T) \in \mathrm{V}\right\}$ is a norm closed subspace of $B(\mathrm{~h})_{*}$, and the set $\left\{\omega_{x, y}: x \in \mathrm{T}^{\prime}, y \in \mathrm{T}\right\}$ is total in $B(\mathrm{~h})_{*}$.

The (right) h-matrix space over $\mathrm{V}$ is the operator space (2.1); we denote it $\mathrm{V} \otimes_{\mathrm{M}} B(\mathrm{~h})$. [Previous notation, used in $\left[\mathrm{LW}_{3}\right]$ and elsewhere: $\mathrm{M}(\mathrm{h} ; \mathrm{V})_{\mathrm{b}}$.]

Remarks. Rectangular matrix spaces $\mathrm{V} \otimes_{\mathrm{M}} B\left(\mathrm{~h} ; \mathrm{h}^{\prime}\right)$ and left matrix spaces $B(\mathrm{~h})_{\mathrm{M}} \otimes \mathrm{V}$ are defined in the obvious way. If $\kappa=\left(e_{i}\right)_{i \in I}$ is an orthonormal basis for $\mathrm{h}$ then, in the notation (1.1), we have the identification

$$
T \in \mathrm{V} \otimes_{\mathrm{M}} B(\mathrm{~h}) \longleftrightarrow{ }^{\kappa} T \in \mathrm{M}_{I}(V)_{\mathrm{b}}
$$

where $\mathrm{M}_{I}(\mathrm{~V})_{\mathrm{b}}$ is defined to be the operator space $\mathrm{M}_{I}(B(\mathrm{H} ; \mathrm{K}))_{\mathrm{b}} \cap \mathrm{M}_{I}(\mathrm{~V})$. Note that $\mathrm{M}_{I}(\mathrm{~V})_{\mathrm{b}}$ has a description valid for abstract matrix spaces $\mathrm{V}$, namely

$$
\left\{A \in \mathrm{M}_{I}(\mathrm{~V}): \sup _{\Lambda \subset \subset I}\left\|A^{[\Lambda]}\right\|<\infty\right\}
$$

where $A^{[\Lambda]} \in \mathrm{M}_{\Lambda}(\mathrm{V})$ denotes the (finite) submatrix of $A$ obtained by cut-off ([EfR], Chapter 10). Significantly, $\mathrm{V} \otimes_{\mathrm{M}} B(\mathrm{~h})$ does too, namely $C B\left(B(\mathrm{~h})_{*} ; \mathrm{V}\right)$ $([\mathrm{LiT}])$.

The following is Lemma 1.1 of $\left[\mathrm{LW}_{3}\right]$, adapted for square-matrix spaces:

Lemma 2.2. Let $\mathrm{V}$ be an operator space in $B(\mathrm{H} ; \mathrm{K})$, and $\mathrm{h}$ and $\mathrm{k}$ any pair of Hilbert spaces. The natural associativity map $B(\mathrm{H} \otimes \mathrm{h} ; \mathrm{K} \otimes \mathrm{h}) \bar{\otimes} B(\mathrm{k}) \rightarrow$ $B(\mathrm{H} ; \mathrm{K}) \bar{\otimes} B(\mathrm{~h} \otimes \mathrm{k})$ restricts to a completely isometric isomorphism

$$
\left(\mathrm{V} \otimes_{\mathrm{M}} B(\mathrm{~h})\right) \otimes_{\mathrm{M}} B(\mathrm{k})=\mathrm{V} \otimes_{\mathrm{M}} B(\mathrm{~h} \otimes \mathrm{k}) .
$$

Diagonal matrix spaces. Let $\mathrm{V}$ be an operator space and $\mathrm{h}$ a Hilbert space with orthonormal basis $\kappa=\left(e_{i}\right)_{i \in I}$. We define the operator space

$$
\mathrm{V} \otimes_{\mathrm{M}} \mathrm{D}_{\kappa}(\mathrm{h}):=\left\{T \in \mathrm{V} \otimes_{\mathrm{M}} B(\mathrm{~h}): E^{(i)} T E_{(j)}=0 \text { for } i \neq j\right\}
$$

(with $E^{(i)}$ and $E_{(j)}$ as in (1.1)) and refer to it as the $\kappa$-diagonal h-matrix space over $\mathrm{V}$. Clearly the completely isometric isomorphism (2.2) restricts 
to a completely isometric isomorphism

$$
\mathrm{V} \otimes_{\mathrm{M}} \mathrm{D}_{\kappa}(\mathrm{h}) \rightarrow \mathrm{D}_{I}(\mathrm{~V})_{\mathrm{b}}
$$

where the diagonal operator subspace of $\mathrm{M}_{I}(\mathrm{~V})_{\mathrm{b}}$ is defined in the obvious way:

$$
\mathrm{D}_{I}(\mathrm{~V})_{\mathrm{b}}:=\left\{\left[a_{j}^{i}\right] \in \mathrm{M}_{I}(\mathrm{~V})_{\mathrm{b}}: a_{j}^{i}=0 \text { for } i \neq j\right\} .
$$

Matrix space liftings. A feature of the matrix space construction that was exploited in $\left[\mathrm{LW}_{3}\right]$ is that completely bounded maps between operator spaces induce completely bounded maps between corresponding matrix spaces. This is detailed in the next result, whose proof follows the same lines as that of Lemma 1.2 of $\left[\mathrm{LW}_{3}\right]$. The result shows in particular that, as operator spaces, matrix spaces do not depend on the concrete representation of the underlying operator space (as already remarked above). In brief, any completely isometric isomorphism between (concrete) operator spaces $\mathrm{V}$ and $\mathrm{W}$ induces a completely isometric isomorphism between $\mathrm{V} \otimes_{\mathrm{M}} B(\mathrm{~h})$ and $\mathrm{W} \otimes_{\mathrm{M}} B(\mathrm{~h})$.

Lemma 2.3. For a completely bounded operator between operator spaces $\phi: \mathrm{V} \rightarrow \mathrm{W}$, and a Hilbert space $\mathrm{h}$, there is a unique map $\phi \otimes_{\mathrm{M}} \mathrm{id}_{B(\mathrm{~h})}$ : $\mathrm{V} \otimes_{\mathrm{M}} B(\mathrm{~h}) \rightarrow \mathrm{W} \otimes_{\mathrm{M}} B(\mathrm{~h})$ satisfying

$$
E^{e}\left(\phi \otimes_{\mathrm{M}} \operatorname{id}_{B(\mathrm{~h})}\right)(T) E_{d}=\phi\left(E^{e} T E_{d}\right) \quad \text { for all } d, e \in \mathrm{h}, T \in \mathrm{V} \otimes_{\mathrm{M}} B(\mathrm{~h}) ;
$$

it is linear and completely bounded, moreover (unless $\mathrm{h}=\{0\}$ ) it satisfies $\left\|\phi \otimes_{\mathrm{M}} \operatorname{id}_{B(\mathrm{~h})}\right\|_{\mathrm{cb}}=\|\phi\|_{\mathrm{cb}}=\left\|\phi \otimes_{\mathrm{M}} \mathrm{id}_{B\left(l^{2}\right)}\right\|$.

Clearly these maps are the coordinate-free counterparts to the sequence of induced maps $\left(\phi^{(n)}\right)_{n \geqslant 1}$. Indeed $\phi \otimes_{\mathrm{M}} \mathrm{id}_{B(\mathrm{~h})}$ is often abbreviated to $\phi^{(\mathrm{h})}$.

Remark. If the operator space $\mathrm{W}$ is of the form $\mathrm{U} \otimes_{\mathrm{M}} B(\mathrm{H})$ then we write

$$
\phi^{\mathrm{h}} \text { for } \Sigma \circ \phi^{(\mathrm{h})}: \mathrm{V} \otimes_{\mathrm{M}} B(\mathrm{~h}) \rightarrow \mathrm{U} \otimes_{\mathrm{M}} B(\mathrm{~h} \otimes \mathrm{H}),
$$

$\Sigma$ being the tensor flip $\mathrm{U} \otimes_{\mathrm{M}} B(\mathrm{H} \otimes \mathrm{h}) \rightarrow \mathrm{U} \otimes_{\mathrm{M}} B(\mathrm{~h} \otimes \mathrm{H})$.

We next address topological questions concerning matrix spaces and induced maps between matrix spaces. For $\omega \in B(\mathrm{~h})_{*}$, we have

$$
\left(\mathrm{id}_{B(\mathrm{H} ; \mathrm{K})} \bar{\otimes} \omega\right)\left(\mathrm{V} \otimes_{\mathrm{M}} B(\mathrm{~h})\right) \subset \mathrm{V} ;
$$

the induced operator $\mathrm{V} \otimes_{\mathrm{M}} B(\mathrm{~h}) \rightarrow \mathrm{V}$ is denoted id $\mathrm{V} \otimes_{\mathrm{M}} \omega$. Let $\phi \in$ $C B(\mathrm{~V} ; \mathrm{W})$ for operator spaces $\mathrm{V}$ and $\mathrm{W}$. The collection of functionals $\omega \in B(\mathrm{~h})_{*}$ satisfying

$$
\left(\mathrm{id}_{\mathrm{W}} \otimes_{\mathrm{M}} \omega\right) \circ\left(\phi \otimes_{\mathrm{M}} \mathrm{id}_{B(\mathrm{~h})}\right)=\phi \circ\left(\mathrm{id} \mathrm{V} \otimes_{\mathrm{M}} \omega\right)
$$

is norm-closed and contains the norm-total family $\left\{\omega_{e, d}: d, e \in \mathrm{h}\right\}$, and so (2.4) holds for all $\omega \in B(\mathrm{~h})_{*}$. Accordingly we denote the resulting map $\mathrm{V} \otimes_{\mathrm{M}} B(\mathrm{~h}) \rightarrow \mathrm{W}$ by $\phi \otimes_{\mathrm{M}} \omega$.

Remark. There is a sense in which a version of identity (2.4) holding for all $\phi$ actually characterises the normality of $\omega$. Precise sufficient conditions are given in [Tom], Theorem 5.1; see also [Neu], Theorem 5.4.

The following result is now evident.

Lemma 2.4. Let $\mathrm{V}$ and $\mathrm{W}$ be operator spaces. 
(a) $\overline{\mathrm{V} \otimes B(\mathrm{~h})}^{\mathrm{h}-w \cdot o .}=\mathrm{V} \otimes_{\mathrm{M}} B(\mathrm{~h})=\overline{\mathrm{V} \otimes B(\mathrm{~h})}^{\mathrm{h}-u w}$.

(b) For a completely bounded operator $\phi: \mathrm{V} \rightarrow \mathrm{W}, \phi \otimes_{\mathrm{M}} \mathrm{id}_{B(\mathrm{~h})}$ is both $\mathrm{h}$-ultraweakly continuous and $\mathrm{h}$-weak operator continuous.

Note the further relations

$$
\mathrm{V} \otimes B(\mathrm{~h}) \subset \mathrm{V} \otimes_{\mathrm{M}} B(\mathrm{~h}) \subset \overline{\mathrm{V}}^{\mathrm{uw}} \bar{\otimes} B(\mathrm{~h}) ;
$$

the first inclusion being an equality if either $\mathrm{V}$ or $\mathrm{h}$ is finite dimensional and the second being an equality if and only if $\mathrm{V}$ is ultraweakly closed. Thus the h-matrix space over an ultraweakly closed operator space is its ultraweak tensor product with $B(\mathrm{~h})$.

Maps with Schur-action. For operator spaces $\mathrm{V}$ and $\mathrm{W}$, index set $I$ and linear map $\phi: \mathrm{M}_{I}(\mathrm{~V})_{\mathrm{b}} \rightarrow \mathrm{M}_{I}(\mathrm{~W})_{\mathrm{b}}$, we can define maps $\phi_{j}^{i}: \mathrm{V} \rightarrow \mathrm{W}$ by

$$
\phi_{j}^{i}(a)=E^{(i)} \phi\left(E_{(i)} a E^{(j)}\right) E_{(j)} .
$$

We say that $\phi$ has Schur-action if these maps determine $\phi$, in the sense that $\phi$ acts componentwise through

$$
\phi\left(\left[a_{j}^{i}\right]\right)=\left[\phi_{j}^{i}\left(a_{j}^{i}\right)\right] .
$$

For example if $\varphi \in C B(\mathrm{~V} ; \mathrm{W})$ and $\mathrm{h}=l^{2}(I)$, then the map $\varphi \otimes_{\mathrm{M}} \mathrm{id}_{B(\mathrm{~h})}$ from Lemma 2.3, viewed as a map $\mathrm{M}_{I}(\mathrm{~V})_{\mathrm{b}} \rightarrow \mathrm{M}_{I}(\mathrm{~W})_{\mathrm{b}}$ (through (2.2)) has Schur-action: $\varphi^{(\mathrm{h})}\left(\left[a_{j}^{i}\right]\right)=\left[\varphi\left(a_{j}^{i}\right)\right]$.

For operator spaces $\mathrm{V}$ and $\mathrm{W}$ and Hilbert space $\mathrm{h}$ with orthonormal basis $\kappa=\left(e_{i}\right)_{i \in I}$, a linear map $\phi: \mathrm{V} \otimes_{\mathrm{M}} B(\mathrm{~h}) \rightarrow \mathrm{W} \otimes_{\mathrm{M}} B(\mathrm{~h})$ will be called $\kappa$-decomposable if the induced map ${ }^{\kappa} \phi: \mathrm{M}_{I}(\mathrm{~V})_{\mathrm{b}} \rightarrow \mathrm{M}_{I}(\mathrm{~W})_{\mathrm{b}}$ given by ${ }^{\kappa} \phi\left({ }^{\kappa} T\right):={ }^{\kappa}(\phi(T))$ (see (1.1)), has Schur-action.

We next establish criteria for a map to have Schur-action. To this end consider the orthogonal projections

$$
p_{k}=\left[\delta_{k}^{i} \delta_{j}^{k} I_{\mathrm{H}}\right]=E_{(k)} E^{(k)}, \quad k \in I,
$$

in $\mathrm{M}_{I}(B(\mathrm{H}))_{\mathrm{b}}$ (for any Hilbert space $\mathrm{H}$ ). The following lemma is easily verified.

Lemma 2.5. Let $\phi$ be a linear map $\mathrm{M}_{I}(\mathrm{~V})_{\mathrm{b}} \rightarrow \mathrm{M}_{I}(\mathrm{~W})_{\mathrm{b}}$ for operator spaces $\mathrm{V}$ and $\mathrm{W}$, and index set $I$. Then the following are equivalent:

(i) $\phi$ has Schur-action.

(ii) $\phi\left(p_{i} A p_{j}\right)=p_{i} \phi(A) p_{j}$ for all $A \in \mathrm{M}_{I}(\mathrm{~V})_{\mathrm{b}}, i, j \in I$.

Specialising to completely positive unital maps between operator systems, Schur-action has the following useful characterisation.

Proposition 2.6. Let $\mathrm{V}$ and $\mathrm{W}$ be operator systems, $I$ an index set, and $\phi$ a linear map $\mathrm{M}_{I}(\mathrm{~V})_{\mathrm{b}} \rightarrow \mathrm{M}_{I}(\mathrm{~W})_{\mathrm{b}}$.

(a) If $\phi$ is unital and has Schur-action then $\phi\left(p_{i}\right)=p_{i}$ for all $i \in I$.

(b) If $\phi\left(p_{i}\right)=p_{i}$ for all $i \in I$ and $\phi$ is a completely positive contraction then $\phi$ is unital and has Schur-action.

Proof. (a) Immediate from Lemma 2.5.

(b) Let $\mathrm{h}=l^{2}(I)$ and identify $\mathrm{M}_{I}(\mathrm{~V})_{\mathrm{b}}$ with $\mathrm{V} \otimes_{\mathrm{M}} B(\mathrm{~h})$. Suppose that $\mathrm{V}$ and $\mathrm{W}$ are operator systems in $B(\mathrm{H})$ and $B(\mathrm{~K})$ respectively, then, by Arveson's Hahn-Banach Theorem ([Pau], Theorem 7.5), $\phi$ extends to a CP 
contraction $\phi^{\prime}: B(\mathrm{H} \otimes \mathrm{h}) \rightarrow B(\mathrm{~K} \otimes \mathrm{h})$. Moreover, $\phi^{\prime}\left(p_{i}^{2}\right)=\phi^{\prime}\left(p_{i}\right)=p_{i}=$ $\phi^{\prime}\left(p_{i}\right)^{2}$, so we have equality in the Kadison-Schwarz inequality and hence, by a result of Choi ([Pau], Proposition 3.18), it follows that

$$
\phi^{\prime}\left(p_{i} T p_{j}\right)=p_{i} \phi^{\prime}(T) p_{j} \quad \text { for all } T \in B(\mathrm{H} \otimes \mathrm{h}), i, j \in I .
$$

In particular for $T \in \mathrm{V} \otimes_{\mathrm{M}} B(\mathrm{~h}), p_{i} T p_{j} \in \mathrm{V} \otimes_{\mathrm{M}} B(\mathrm{~h})$ and so $\phi$ has Schuraction by Lemma 2.5. Finally, $\phi$ is unital since for all $i, j \in I$

$$
p_{i} \phi(I) p_{j}=\phi\left(p_{i} p_{j}\right)=\delta_{j}^{i} \phi\left(p_{i}\right)=\delta_{j}^{i} p_{i}=p_{i} I p_{j} .
$$

Remarks. (i) Choi's result is stated in [Pau] for unital maps, but the proof shows that contractivity suffices.

(ii) In the absence of a normality assumption on $\phi$, the following example shows that the contractivity assumption in (b) is needed. Let $\mathrm{h}=l^{2}(\mathbb{N})$, let $\mathcal{K}$ denote the algebra of compact operators on $\mathrm{h}$ and let $\mathrm{V}=\mathrm{W}=\mathbb{C}$, so that $\mathrm{M}_{\mathbb{N}}(\mathrm{V})_{\mathrm{b}} \cong B(\mathrm{~h})$, and let $\varphi=\mathrm{id}_{\mathcal{K}}$. For $\lambda>1$ define a $\mathrm{CP}$ extension of $\varphi$ to $\mathcal{K}+\mathbb{C} I$ by

$$
\varphi^{\lambda}(T+\mu I)=T+\lambda \mu I
$$

using Proposition 1.3, and extend this to a CP map $\Phi^{\lambda}$ on $B(\mathrm{~h})$ by Arveson's Hahn-Banach Theorem. Then $\Phi^{\lambda}\left(p_{i}\right)=p_{i}$ but $\Phi^{\lambda}(I)=\lambda I \neq I$.

\section{Quantum stochastic Processes}

Let $\mathrm{k}$ be a fixed but arbitrary complex Hilbert space, referred to at the noise dimension space. The orthogonal sum $\mathbb{C} \oplus \mathrm{k}$ is denoted $\widehat{k}$.

Fock space. The symmetric Fock space over $L^{2}\left(\mathbb{R}_{+} ; \mathrm{k}\right)$ is denoted $\mathcal{F}$. We use normalised exponential vectors (also called coherent vectors)

$$
\varpi(f):=e^{-\frac{1}{2}\|f\|^{2}}\left((n !)^{-1 / 2} f^{\otimes n}\right)_{n \geqslant 0}, f \in L^{2}\left(\mathbb{R}_{+} ; \mathbf{k}\right) ;
$$

these are linearly independent and satisfy $\langle\varpi(f), \varpi(g)\rangle=\exp (-\chi(f, g))$, where, for any Hilbert space $\mathrm{H}$,

$$
\chi(u, v):=\frac{1}{2}\left(\|u\|^{2}+\|v\|^{2}\right)-\langle u, v\rangle, \quad u, v \in \mathrm{H} .
$$

For a subset $\mathrm{T}$ of $\mathrm{k}$ we set

$$
\mathcal{E}_{\boldsymbol{\top}}:=\operatorname{Lin}\left\{\varpi(f): f \in \mathbb{S}_{\boldsymbol{\top}}\right\},
$$

where $\mathbb{S}_{T}$ denotes the collection of (right-continuous) T-valued step functions in $L^{2}\left(\mathbb{R}_{+} ; \mathrm{k}\right.$ ), and abbreviate to $\mathcal{E}$ and $\mathbb{S}$ when $\mathrm{T}$ is all of $\mathrm{k}$ (note that necessarily $0 \in \mathrm{T}$ ). Then $\mathcal{E}_{\mathrm{T}}$ is dense in $\mathcal{F}$ if and only if $\mathrm{T}$ is total in k. For example $\mathbf{T}=\{0\} \cup\left\{d_{i}: i \in I_{0}\right\}$ for an orthonormal basis $\left\{d_{i}\right\}_{i \in I_{0}}$ for k. For a proof of this result, which is due to Parthasarathy and Sunder, and Skeide, and the basics of quantum stochastics, we refer to [L].

For each subinterval $J$ of $\mathbb{R}_{+}$we denote the symmetric Fock space over $L^{2}\left(J ; \mathbb{R}_{+}\right)$by $\mathcal{F}_{J}$, and the identity operator on $\mathcal{F}_{J}$ by $I_{J}$. Then the natural identifications

$$
\mathcal{F}=\mathcal{F}_{[0, r[} \otimes \mathcal{F}_{[r, t]} \otimes \mathcal{F}_{[t, \infty[}, \quad 0 \leqslant r \leqslant t \leqslant \infty,
$$

are effected by

$$
\varpi(f) \mapsto \varpi\left(\left.f\right|_{[0, r[}\right) \otimes \varpi\left(\left.f\right|_{[r, t]}\right) \otimes \varpi\left(\left.f\right|_{[t, \infty[}\right),
$$

and $\mathcal{F}_{[0, t[}$ is naturally isometric to the subspace $\mathcal{F}_{[0, t[} \otimes \varpi\left(\left.0\right|_{[t, \infty[}\right)$ of $\mathcal{F}$. 
By this means we make the identifications

$$
B(\mathcal{F})=B\left(\mathcal{F}_{[0, r[}\right) \bar{\otimes} B\left(\mathcal{F}_{[r, t}\right] \bar{\otimes} B\left(\mathcal{F}_{[t, \infty[}\right)
$$

and, in turn,

$$
B\left(\mathcal{F}_{[r, t]}\right)=I_{[0, r[} \otimes B\left(\mathcal{F}_{[r, t[}\right) \otimes I_{[t, \infty[} \subset B(\mathcal{F}) .
$$

The Fock-Weyl operators may be defined on $\mathcal{F}$ by continuous linear extension of the prescription

$$
W(f): \varpi(g) \mapsto e^{-i \operatorname{Im}\langle f, g\rangle} \varpi(f+g), \quad f, g \in L^{2}\left(\mathbb{R}_{+} ; \mathbf{k}\right) .
$$

These are unitary operators satisfying the canonical commutation relations in exponential/Weyl form:

$$
W(f) W(g)=e^{-i \operatorname{Im}\langle f, g\rangle} W(f+g) .
$$

Note that the probabilistic normalisation $([\mathrm{L}]$, [Mey], $[\mathrm{Par}])$ is preferred here rather than the usual quantum theoretic one ([BrR]).

We next record a notation which will be heavily used in the sequel:

$$
E(f):=E_{\varpi(f)}, \quad f \in L^{2}\left(\mathbb{R}_{+} ; \mathrm{k}\right) .
$$

Thus $E(f)=(I \otimes W(f)) E_{\varpi(0)}$.

The $C C R$ flow on $B(\mathcal{F})$ is the one-parameter semigroup of normal, unital, *-endomorphisms determined by

$$
\sigma_{t}^{\mathrm{k}}(W(f))=W\left(s_{t} f\right)
$$

where $\left(s_{t}\right)_{t \geqslant 0}$ is the semigroup of right shifts on $L^{2}\left(\mathbb{R}_{+} ; \mathrm{k}\right)$. Thus $\sigma_{t}^{\mathrm{k}}(B(\mathcal{F}))=$ $I_{[0, t[} \otimes B\left(\mathcal{F}_{[t, \infty[}\right)$.

Processes. Let $\mathrm{V}$ be an operator space in $B\left(\mathfrak{h} ; \mathfrak{h}^{\prime}\right)$. A bounded quantum stochastic process in $\mathrm{V}$ (with noise dimension space $\mathrm{k}$ ) is a family of operators $\left(X_{t}\right)_{t \geqslant 0}$ satisfying the adaptedness condition

$$
X_{t} \in \mathrm{V} \otimes_{\mathrm{M}} B\left(\mathcal{F}_{[0, t]}\right) \otimes I_{[t, \infty[} \text { for all } t \in \mathbb{R}_{+} .
$$

In practice a weak measurability condition is also imposed; however in this paper such an assumption plays no role. When $\mathrm{V}=B(\mathfrak{h})$ we speak of a bounded $Q S$ process on $\mathfrak{h}$. The self-adjoint unitary process $R^{\mathrm{k}}$ defined by

$$
R_{t}^{\mathrm{k}} \varpi(f)=\varpi\left(r_{t} f\right) \text { where }\left(r_{t} f\right)(s)= \begin{cases}f(t-s) & \text { if } s \in[0, t[, \\ f(s) & \text { if } s \in[t, \infty[,\end{cases}
$$

plays a fundamental role. For a bounded process $X$ on $\mathfrak{h}$ the time-reversed process is defined by

$$
X^{R}:=\left(R_{t} X_{t} R_{t}\right)_{t \geq 0} \quad \text { where } R_{t}=I_{\mathfrak{h}} \otimes R_{t}^{\mathrm{k}} .
$$

We are primarily interested in bounded QS processes on an operator space $\mathrm{V}$. These are families of bounded operators $k_{t}: \mathrm{V} \rightarrow \mathrm{V} \otimes_{\mathrm{M}} B(\mathcal{F})$ such that $\left(k_{t}(a)\right)_{t \geqslant 0}$ is a process in $\mathrm{V}$ for each $a \in \mathrm{V}$. Such a process is called completely bounded, completely contractive, or (when $\mathrm{V}$ is an operator system or $C^{*}$ algebra) completely positive, if each $k_{t}$ is. The property $k_{t}(\mathrm{~V}) \subset \mathrm{V} \otimes_{\mathrm{M}} B(\mathcal{F})$ is a noncommutative form of Feller condition (see $\left.\left[\mathrm{LW}_{3}\right]\right)$. 


\section{Completely Bounded Quantum Stochastic COCyCles}

Let $\vee$ be an operator space in $B\left(\mathfrak{h} ; \mathfrak{h}^{\prime}\right)$. A completely bounded QS process $k$ on $\mathrm{V}$ is called a quantum stochastic cocycle on $\mathrm{V}$ if it satisfies

$$
k_{0}=\iota_{\mathcal{F}} \text { and } k_{r+t}=\widehat{k}_{r} \circ \sigma_{r} \circ k_{t} \text { for } r, t \in \mathbb{R}_{+},
$$

where $\iota_{\mathcal{F}}$ denotes the ampliation $a \mapsto a \otimes I_{\mathcal{F}}, \sigma_{r}$ is the shift obtained by restriction to $\mathrm{V} \otimes_{\mathrm{M}} B(\mathcal{F})$ of the $\operatorname{map~id}_{B\left(\mathfrak{h} ; \mathfrak{h}^{\prime}\right)} \bar{\otimes} \sigma_{r}^{\mathrm{k}}$, and $\widehat{k}_{r}:=k_{r} \otimes_{\mathrm{M}} \operatorname{id}_{B\left(\mathcal{F}_{[r, \infty[}\right)}$. For the lifting $\widehat{k}_{r}$, the following identifications are invoked:

$\operatorname{Ran} \sigma_{r}=\mathrm{V} \otimes_{\mathrm{M}} B\left(\mathcal{F}_{[r, \infty[}\right)$ and $\mathrm{V} \otimes_{\mathrm{M}} B\left(\mathcal{F}_{[0, r[}\right) \otimes_{\mathrm{M}} B\left(\mathcal{F}_{[r, \infty[}\right)=\mathrm{V} \otimes_{\mathrm{M}} B(\mathcal{F})$

In this paper, all QS cocycles on an operator space will be assumed to be completely bounded.

To each bounded process $k$ we associate the family of bounded operators $k_{t}^{f, g}: \mathrm{V} \rightarrow \mathrm{V}$, indexed by ordered pairs $(f, g)$ from $L_{\text {loc }}^{2}\left(\mathbb{R}_{+} ; \mathrm{k}\right)$, defined, in the notation (3.2), by

$$
k_{t}^{f, g}(a)=E\left(f_{[0, t]}\right)^{*} k_{t}(a) E\left(g_{[0, t]}\right) .
$$

Remarks. (i) Unnormalised exponential vectors were used for defining the maps $k_{t}^{f, g}$ in earlier papers $\left.\left(\left[\mathrm{LW}_{2}\right],\left[\mathrm{LW}_{3}\right]\right]\right)$. Benefits of normalising will be seen later.

(ii) We identify the noise dimension space $\mathrm{k}$ with the constant functions in $L_{\text {loc }}^{2}\left(\mathbb{R}_{+} ; \mathrm{k}\right)$.

The proposition below, in the context of von Neumann algebras and normal completely bounded operators, goes back to $\left[\mathrm{LW}_{2}\right]$.

Proposition 4.1. Let $k$ be a completely bounded process on an operator space $\mathrm{V}$ in $B\left(\mathfrak{h} ; \mathfrak{h}^{\prime}\right)$, set $\mathcal{P}_{t}^{x, y}:=k_{t}^{x, y}(x, y \in \mathrm{k}, t \geqslant 0)$ and let $\mathrm{T}$ and $\mathrm{T}^{\prime}$ be total subsets of $\mathrm{k}$ containing 0 . Then the following are equivalent:

(i) $k$ is a $Q S$ cocycle on $\mathrm{V}$;

(ii) $k_{0}^{f, g}=\mathrm{id} \vee$ and $k_{r+t}^{f, g}=k_{r}^{f, g} \circ k_{t}^{s_{r}^{*} f, s_{r}^{*} g}$ for all $f \in \mathbb{S}_{\mathrm{T}}, g \in \mathbb{S}_{\boldsymbol{T}^{\prime}}$ and $r, t \geqslant 0$.

(iii) For all $x \in \mathrm{T}$ and $y \in \mathrm{T}^{\prime},\left(\mathcal{P}_{t}^{x, y}\right)_{t \geqslant 0}$ defines a semigroup on $\mathrm{V}$, and for all $f \in \mathbb{S}_{\boldsymbol{\top}}, g \in \mathbb{S}_{\boldsymbol{T}^{\prime}}$ and $t>0$

$$
k_{t}^{f, g}=\mathcal{P}_{t_{1}-t_{0}}^{x_{0}, y_{0}} \circ \cdots \circ \mathcal{P}_{t_{m+1}-t_{m}}^{x_{m}, y_{m}},
$$

where $t_{0}=0, t_{m+1}=t,\left\{t_{1}<\cdots<t_{m}\right\}$ is the (possibly empty) union of the sets of points of discontinuity of $f$ and $g$ in $] 0, t[$ and, for $i=0, \ldots, m, x_{i}:=f\left(t_{i}\right)$ and $y_{i}:=g\left(t_{i}\right)$.

(iv) For all $f \in \mathbb{S}_{\mathrm{T}}, g \in \mathbb{S}_{\mathrm{T}^{\prime}}$ and $t \geqslant 0, k_{0}^{f, g}=\mathrm{id} \vee$ and, whenever $\{0=$ $\left.s_{0} \leqslant s_{1} \leqslant \ldots \leqslant s_{n+1}=t\right\}$ contains all the points of discontinuity of $f_{[0, t[}$ and $g_{[0, t[}$,

$$
k_{t}^{f, g}=\mathcal{P}_{s_{1}-s_{0}}^{x_{0}, y_{0}} \circ \cdots \circ \mathcal{P}_{s_{n+1}-s_{n}}^{x_{n}, y_{n}}
$$

where, for $j=0, \ldots, n, x_{j}:=f\left(s_{j}\right)$ and $y_{j}:=g\left(s_{j}\right)$. 
Proof. Let $r, s \in \mathbb{R}_{+}$with $r \leqslant s$. The following identities, in which $h \in$ $L^{2}\left(\mathbb{R}_{+} ; \mathrm{k}\right), T \in \mathrm{V} \otimes_{\mathrm{M}} B\left(\mathcal{F}_{[r, \infty[}\right)$ and $X \in B\left(\mathfrak{h} ; \mathfrak{h}^{\prime}\right) \bar{\otimes} B(\mathcal{F})$, are straightforward consequences of the definitions:

$$
\begin{aligned}
& E(h)=E\left(h_{[r, \infty[}\right) E\left(h_{[0, r]}\right), \\
& E\left(f_{[r, \infty[}\right)^{*} \widehat{k}_{r}(T) E\left(g_{[r, \infty[}\right)=k_{r}\left(E\left(f_{[r, \infty[}\right)^{*} T E\left(g_{[r, \infty[}\right)\right), \\
& E\left(f_{[r, \infty[}\right)^{*} \sigma_{r}(X) E\left(g_{[r, \infty[}\right)=E\left(s_{r}^{*} f\right)^{*} X E\left(s_{r}^{*} g\right),
\end{aligned}
$$

as is the inclusion

$$
\widehat{k}_{r}\left(\mathrm{~V} \otimes_{\mathrm{M}} B\left(\mathcal{F}_{[r, s[}\right) \otimes I_{[s, \infty[}\right) \subset \mathrm{V} \otimes_{\mathrm{M}} B\left(\mathcal{F}_{[0, s]}\right) \otimes I_{[s, \infty[}
$$

Therefore, for $a \in V$ and $r, t \in \mathbb{R}_{+}$,

$$
\begin{aligned}
E\left(f_{[0, r+t]}\right)^{*}\left(\widehat{k}_{r} \circ\right. & \left.\sigma_{r} \circ k_{t}\right)(a) E\left(g_{[0, r+t]}\right) \\
& =E\left(f_{[0, r[}\right)^{*} E\left(f_{[r, r+t]} \widehat{k}_{r}\left(\left(\sigma_{r} \circ k_{t}\right)(a)\right) E\left(g_{[r, r+t]}\right) E\left(g_{[0, r[}\right)\right. \\
& =E\left(f_{[0, r[}\right)^{*} k_{r}\left(E\left(s_{r}^{*} f_{[0, r+t[}\right) k_{t}(a) E\left(s_{r}^{*} g_{[0, r+t[}\right)\right) E\left(g_{[0, r[}\right) \\
& =k_{r}^{f, g} \circ k_{t}^{s_{r}^{*} f, s_{r}^{*} g}(a),
\end{aligned}
$$

since $\left(\widehat{k}_{r} \circ \sigma_{r} \circ k_{t}\right)(a) \in \mathrm{V} \otimes_{\mathrm{M}} B\left(\mathcal{F}_{[0, r+t[}\right)$, and so the equivalence of (i) and (ii) follows from the totality of $\mathcal{E}_{\mathrm{T}}$ and $\mathcal{E}_{\mathrm{T}^{\prime}}$ in $\mathcal{F}$. The equivalence of (ii), (iii) and (iv) follows from the fact that $s_{u}^{*} z=z$ for all $z \in \mathrm{k}$ and $u \in \mathbb{R}_{+}$.

We refer to $\left\{\mathcal{P}^{x, y}: x, y \in \mathrm{k}\right\}$ as the associated semigroups of the cocycle, $\mathcal{P}^{0,0}$ as its (vacuum) expectation semigroup, and (4.2) or (4.3) as the semigroup decomposition/characterisation for QS cocycles. Note that if the cocycle $k$ is contractive then so are each of its associated semigroups. The following is an immediate consequence of the above.

Corollary 4.2. Let $j$ and $k$ be completely bounded $Q S$ cocycles on an operator space, with respective associated semigroups $\left\{\mathcal{P}^{x, y}: x, y \in \mathrm{k}\right\}$ and $\left\{\mathcal{Q}^{x, y}: x, y \in \mathrm{k}\right\}$, and let $\mathrm{T}$ and $\mathrm{T}^{\prime}$ be total subsets of $\mathrm{k}$ containing 0 . Then $j=k$ if and only if $\mathcal{P}^{x, y}=\mathcal{Q}^{x, y}$ for all $x \in \mathrm{T}^{\prime}$ and $y \in \mathrm{T}$.

Notation. If $\left\{\mathcal{R}^{x, y}: x, y \in \mathrm{T}\right\}$ is an indexed family of linear maps on the operator space $\mathrm{V}$ then, for $n \geqslant 1$ and $\mathbf{x} \in \mathrm{T}^{n}$, we write $\mathcal{R}^{\mathbf{x}}$ for the Schuraction map on $\mathrm{M}_{n}(\mathrm{~V})$ with component maps $\left\{\mathcal{R}^{x_{i}, x_{j}}: i, j=1, \ldots, n\right\}$ :

$$
\mathcal{R}^{\mathbf{x}}\left(\left[u_{j}^{i}\right]\right)=\left[\mathcal{R}^{x_{i}, x_{j}}\left(u_{j}^{i}\right)\right]
$$

In conjunction with the representation (4.8) below, the following result is useful for extracting positivity and contractivity properties of QS cocycles.

Proposition 4.3. Let $k$ be a $Q S$ cocycle on an operator space $\mathrm{V}$ with associated semigroups $\left\{\mathcal{P}^{x, y}: x, y \in \mathbf{k}\right\}$, and let $\mathbf{f} \in \mathbb{S}^{N}$. Then $k_{t}^{\mathbf{f}}$, the Schur-action map on $\mathrm{M}_{N}(\mathrm{~V})$ with components $\left\{k_{t}^{f_{i}, f_{j}}: i, j=1, \ldots, N\right\}$, satisfies

$$
k_{t}^{\mathbf{f}}=\mathcal{P}_{t_{1}-t_{0}}^{\mathbf{x}(0)} \circ \cdots \circ \mathcal{P}_{t_{n+1}-t_{n}}^{\mathbf{x}(n)}, \text { where } \mathbf{x}(k):=\mathbf{f}\left(t_{k}\right),
$$

whenever $\left\{0=t_{0} \leqslant \cdots \leqslant t_{n+1}=t\right\}$ contains the discontinuities of $\mathbf{f}_{[0, t[}$.

Proof. Since each semigroup $\mathcal{P}^{\mathbf{x}(k)}$ has Schur-action, the result follows immediately from Proposition 4.1. 
Matrices and liftings of cocycles. For $i, j \in\{1, \ldots, n\}$ let $\mathrm{V}_{j}^{i}$ be an operator space in $B\left(\mathfrak{h}_{i} ; \mathfrak{h}_{j}^{\prime}\right)$ and let $k_{j}^{i}$ be a $\mathrm{QS}$ cocycle on $\mathrm{V}_{j}^{i}$. Then the Schur-action

$$
k_{t}\left(\left[a_{j}^{i}\right]\right):=\left[k_{j}^{i}(t)\left(a_{j}^{i}\right)\right]
$$

defines a QS cocycle on the operator space

$$
\left.\mathrm{V}:=\left\{\left[a_{j}^{i}\right] \in B\left(\bigoplus_{i} \mathfrak{h}_{i} ; \bigoplus_{j} \mathfrak{h}_{j}^{\prime}\right)\right): a_{j}^{i} \in \mathrm{V}_{j}^{i} \text { for all } i, j\right\} .
$$

This follows from the identity

$$
k_{t}^{f, g}\left(\left[a_{j}^{i}\right]\right)=\left[k_{j}^{i}(t)^{f, g}\left(a_{j}^{i}\right)\right],
$$

for $f, g \in L_{\text {loc }}^{2}\left(\mathbb{R}_{+} ; \mathrm{k}\right)$.

As an example let $k$ be a QS cocycle on an operator space $\vee$ in $B\left(\mathfrak{h} ; \mathfrak{h}^{\prime}\right)$, then $k^{\dagger}$ is a cocycle on $\mathrm{V}^{\dagger}$ with $\left(k_{t}^{\dagger}\right)^{f, g}=\left(k_{t}^{g, f}\right)^{\dagger}$ and so, recalling (1.4) and (1.5), $\widetilde{k}$ is a cocycle on the operator system $\widetilde{V}$. The associated semigroups of $\widetilde{k}$ are given by

$$
\widetilde{\mathcal{P}}_{t}^{x, y}\left(\left[\begin{array}{cc}
\alpha I_{\mathfrak{h}^{\prime}} & a \\
b & \beta I_{\mathfrak{h}}
\end{array}\right]\right)=\left[\begin{array}{cc}
\alpha e^{-t \chi(x, y)} I_{\mathfrak{h}^{\prime}} & \mathcal{P}_{t}^{x, y}(a) \\
\left(\mathcal{P}_{t}^{y, x}\right)^{\dagger}(b) & \beta e^{-t \chi(x, y)} I_{\mathfrak{h}}
\end{array}\right] ;
$$

where $\left\{\mathcal{P}^{x, y}: x, y \in \mathrm{k}\right\}$ are the associated semigroups of $k$. The identification of $\mathrm{M}_{n}(\widetilde{\mathrm{V}})$ given in (1.6) entails the action

$$
\widetilde{\mathcal{P}}_{t}^{\mathbf{x}}:\left[\begin{array}{cc}
I_{\mathfrak{h}^{\prime}} \otimes \lambda & A \\
B & I_{\mathfrak{h}} \otimes \mu
\end{array}\right] \mapsto\left[\begin{array}{cc}
I_{\mathfrak{h}^{\prime}} \otimes\left(\lambda \cdot \varpi_{t}^{\mathbf{x}}\right) & \mathcal{P}_{t}^{\mathbf{x}}(A) \\
\left(\mathcal{P}_{t}^{\mathbf{x}}\right)^{\dagger}(B) & I_{\mathfrak{h}} \otimes\left(\mu \cdot \varpi_{t}^{\mathbf{x}}\right)
\end{array}\right]
$$

where for each $n \geqslant 1, \mathbf{x} \in \mathrm{k}^{n}$ and $t \geqslant 0$, we define the Grammian matrix

$$
\left.\varpi_{t}^{\mathbf{x}}:=\left[\left\langle\varpi\left(x_{[0, t]}^{i}\right), \varpi\left(x_{[0, t]}^{j}\right)\right\rangle\right]=\left[e^{-t \chi\left(x^{i}, x^{j}\right.}\right)\right] \in \mathrm{M}_{n}(\mathbb{C})_{+},
$$

and $\cdot$ denotes the Schur product of scalar matrices.

Warning. The scope of the tilde is important: in general

$$
\left[\begin{array}{cc}
\alpha e^{-t \chi(x, y)} I_{\mathfrak{h}^{\prime}} & \mathcal{P}_{t}^{x, y}(a) \\
\left(\mathcal{P}_{t}^{y, x}\right)^{\dagger}(b) & \beta e^{-t \chi(x, y)} I_{\mathfrak{h}}
\end{array}\right] \neq\left[\begin{array}{cc}
\alpha I_{\mathfrak{h}^{\prime}} & \mathcal{P}_{t}^{x, y}(a) \\
\mathcal{P}_{t}^{x, y}\left(b^{*}\right)^{*} & \beta I_{\mathfrak{h}}
\end{array}\right]
$$

so $\widetilde{\mathcal{P}}_{t}^{x, y} \neq \widetilde{\mathcal{P}_{t}^{x, y}}$.

The following identity is useful for the examination of properties of a QS cocycle $k$ on an operator space $\mathrm{V}$ in $B\left(\mathfrak{h} ; \mathfrak{h}^{\prime}\right)$. If $\xi \in(\mathfrak{h} \otimes \mathcal{E})^{n}$ with representation $\xi^{i}=\sum_{p=1}^{N} u_{p}^{i} \otimes \varpi\left(f_{p}^{i}\right), i=1, \ldots, n$, and $\xi^{\prime} \in\left(\mathfrak{h}^{\prime} \underline{\otimes} \mathcal{E}\right)^{n}$ with corresponding 'primed' representation then, for each $A \in \mathrm{M}_{n}(\mathrm{~V})$,

$$
\left\langle\xi^{\prime}, k_{t}^{(n)}(A) \xi\right\rangle=\left\langle\eta^{\prime}, k_{t}^{(n N)}\left(A \otimes \square_{N}\right) \eta\right\rangle
$$

where

$$
\square_{N}:=\left[\begin{array}{cccc}
1 & 1 & \cdots & 1 \\
1 & 1 & \cdots & 1 \\
\vdots & \vdots & \ddots & \vdots \\
1 & 1 & \cdots & 1
\end{array}\right] \in \mathrm{M}_{N}(\mathbb{C}) \text { and } \eta=\left(\begin{array}{c}
\eta_{1} \\
\vdots \\
\eta_{N}
\end{array}\right) \in\left((\mathfrak{h} \underline{\otimes} \mathcal{E})^{n}\right)^{N}
$$

with $\eta_{p}^{i}=u_{p}^{i} \otimes \varpi\left(f_{p}^{i}\right)$, and $\eta^{\prime}$ defined correspondingly.

Another useful construction of new cocycles from old is obtained by lifting, as follows. Recall the notation (2.3). If $k$ is a QS cocycle on an operator space $\mathrm{V}$ then, for any Hilbert space $\mathrm{h}, k^{\mathrm{h}}:=\left(k_{t}^{\mathrm{h}}\right)_{t \geqslant 0}$ defines a QS cocycle on 
$\mathrm{V} \otimes_{\mathrm{M}} B(\mathrm{~h})$. As an immediate application we extend a fundamental estimate for $C_{0}$-semigroups to QS cocycles.

Proposition 4.4. Let $k$ be a $Q S$ cocycle on an operator space $\mathrm{V}$, with locally bounded $C B$ norm. Then there exist constants $M \geqslant 1$ and $\omega \in \mathbb{R}$ such that

$$
\left\|k_{t}\right\|_{\mathrm{cb}} \leqslant M e^{\omega t} \quad \text { for all } t \geqslant 0 .
$$

Proof. Let $\mathrm{h}$ be any infinite dimensional Hilbert space. Then by the cocycle identity for $k^{\mathrm{h}}$, and the complete isometry of the shifts,

$$
\left\|k_{r+t}\right\|_{\mathrm{cb}}=\left\|k_{r+t}^{\mathrm{h}}\right\| \leqslant\left\|\widehat{k_{r}^{\mathrm{h}}}\right\|\left\|k_{t}^{\mathrm{h}}\right\|=\left\|k_{r}\right\|_{\mathrm{cb}}\left\|k_{t}\right\|_{\mathrm{cb}}
$$

The result therefore follows by standard arguments from semigroup theory (e.g. [Dav], Lemma 1.2.1).

Operator QS cocycles. In $[\mathrm{LiP}],\left[\mathrm{LW}_{1}\right]$ and $\left[\mathrm{LW}_{2}\right]$ properties of an operator process on $\mathfrak{h}$ are deduced from results concerning QS cocycles, flows and QS differential equations on operator algebras, by constructing an associated process on $B(\mathfrak{h})$. However, full algebras are not necessarily the best choice, as is shown in Theorem 5.8 below. More significantly the tools of operator space theory - in particular Proposition 1.4 - provide an alternative means of seeing the two types of process from a common viewpoint facilitating further analysis.

A bounded process $X$ on $\mathfrak{h}$ is a left (resp. right) quantum stochastic cocycle if $X_{0}=I_{\mathfrak{h} \otimes \mathcal{F}}$ and

$$
X_{r+t}=X_{r} \sigma_{r}\left(X_{t}\right) \quad\left(\text { resp. } X_{r+t}=\sigma_{r}\left(X_{t}\right) X_{r}\right) \quad \text { for all } r, t \geqslant 0 .
$$

Thus $X$ is a left cocycle on $\mathfrak{h}$ if and only if $X^{*}:=\left(X_{s}^{*}\right)_{s \geqslant 0}$ is a right cocycle on $\mathfrak{h}$.

Proposition 4.5. For bounded $Q S$ processes $X$ and $Y$ on $\mathfrak{h}$ define completely bounded $Q S$ processes ${ }^{1} k$ on $|\mathfrak{h}\rangle,{ }^{2} k$ on $\langle\mathfrak{h}|$, and ${ }^{3} k$ and ${ }^{4} k$ on $B(\mathfrak{h})$ by

$$
\begin{aligned}
{ }^{1} k_{s}(|u\rangle) & =X_{s}\left(|u\rangle \otimes I_{\mathcal{F}}\right), & & { }^{3} k_{s}(a)=X_{s}\left(a \otimes I_{\mathcal{F}}\right) X_{s}^{*}, \quad \text { and } \\
{ }^{2} k_{s}(\langle u|) & =\left(\langle u| \otimes I_{\mathcal{F}}\right) Y_{s}, & & { }^{4} k_{s}(a)=Y_{s}^{*}\left(a \otimes I_{\mathcal{F}}\right) Y_{s},
\end{aligned}
$$

for $u \in \mathfrak{h}$ and $a \in B(\mathfrak{h})$.

(a) ${ }^{1} k$ is a cocycle on $|\mathfrak{h}\rangle$ if and only if $X$ is a left cocycle on $\mathfrak{h}$, in which case ${ }^{3} k$ is a cocycle on $B(\mathfrak{h})$.

(b) ${ }^{2} k$ is a cocycle on $\langle\mathfrak{h}|$ if and only if $Y$ is a right cocycle on $\mathfrak{h}$, in which case ${ }^{4} k$ is a cocycle on $B(\mathfrak{h})$.

Furthermore, $\left\|X_{s}\right\|=\left\|{ }^{1} k_{s}\right\|_{\mathrm{cb}}=\left\|{ }^{3} k_{s}\right\|=\left\|{ }^{3} k_{s}\right\|_{\mathrm{cb}}$; similarly for $Y,{ }^{2} k$ and ${ }^{4} k$.

Proof. Adaptedness of the process $X$ amounts to the statement: for all $t \geqslant 0$, $v \in \mathfrak{h}$ and $f \in L^{2}\left(\left[0, t[; \mathrm{k})\right.\right.$ there is $\xi \in \mathfrak{h} \otimes \mathcal{F}_{[0, t[}$ such that

$$
X_{t}(v \otimes \varpi(f) \otimes \varpi(g))=\xi \otimes \varpi(g) \quad\left(g \in L^{2}([t, \infty[; \mathbf{k})) .\right.
$$

Similarly for ${ }^{1} k$ and ${ }^{3} k$ with the left hand side replaced by ${ }^{1} k_{t}(|v\rangle) \varpi(f)$ or $3 k_{t}(a) v \otimes \varpi(f)$. The implications in (a) then follow from the easily verified 
relations

$$
\begin{aligned}
& \widehat{1}_{s} \circ \sigma_{s} \circ{ }^{1} k_{t}(|u\rangle)=X_{s} \sigma_{s}\left(X_{t}\right)\left(|u\rangle \otimes I_{\mathcal{F}}\right) ; \\
& \widehat{3}_{r} \circ \sigma_{r} \circ{ }^{3} k_{t}(a)=X_{r} \sigma_{r}\left(X_{t}\right)\left(a \otimes I_{\mathcal{F}}\right) \sigma_{s}\left(X_{t}\right)^{*} X_{r}^{*},
\end{aligned}
$$

and (b) follows by taking adjoints, since ${ }^{1} k_{s}^{\dagger}={ }^{2} k_{s}$ if $Y=X^{*}$. The norm identities are immediate.

Thus the natural completely isometric isomorphism between $B(\mathfrak{h}) \bar{\otimes} B(\mathcal{F})$ and $C B(|\mathfrak{h}\rangle ;|\mathfrak{h}\rangle \bar{\otimes} B(\mathcal{F})$ ) (resp. $C B(\langle\mathfrak{h}| ;\langle\mathfrak{h}| \bar{\otimes} B(\mathcal{F}))$ ) interchanges processes on $\mathfrak{h}$ with processes on $|\mathfrak{h}\rangle$ (resp. $\langle\mathfrak{h}|)$ and left (resp. right) QS cocycles on $\mathfrak{h}$ with QS cocycles on $|\mathfrak{h}\rangle$ (resp. $\langle\mathfrak{h}|)$. Note also the relations

$$
{ }^{1} k_{s}^{f, g}(|u\rangle)=X_{s}^{f, g}|u\rangle \text { and }{ }^{2} k_{s}^{f, g}(\langle u|)=\langle u| Y_{s}^{f, g},
$$

where $X_{s}^{f, g}$ is defined analogously to (4.1). These identities and Proposition 4.1 yields the following:

Proposition 4.6. Let $X$ be a bounded $Q S$ process on $\mathfrak{h}$, set $P_{t}^{x, y}:=X_{t}^{x, y}$ $(x, y \in \mathrm{k}, t \geqslant 0)$, and let $\mathrm{T}$ and $\mathrm{T}^{\prime}$ be total subsets of $\mathrm{k}$ containing 0 . Then the following are equivalent:

(i) $X$ is a left (respectively right) $Q S$ cocycle on $\mathfrak{h}$.

(ii) $X_{0}^{f, g}=I_{\mathfrak{h}}$ and $X_{r+t}^{f, g}=X_{r}^{f, g} X_{t}^{s_{r}^{*} f, s_{r}^{*} g}$ (resp. $\left.X_{r+t}^{f, g}=X_{t}^{s_{r}^{*} f, s_{r}^{*} g} X_{r}^{f, g}\right)$ for all $f \in \mathbb{S}_{\mathrm{T}}, g \in \mathbb{S}_{\boldsymbol{T}^{\prime}}$ and $r, t \geqslant 0$.

(iii) For all $x \in \mathrm{T}$ and $y \in \mathrm{T}^{\prime},\left(P_{t}^{x, y}\right)_{t \geqslant 0}$ defines a semigroup on $\mathfrak{h}$, and for all $f \in \mathbb{S}_{\boldsymbol{\top}}, g \in \mathbb{S}_{\boldsymbol{T}^{\prime}}$ and $t>0$

$$
X_{t}^{f, g}=P_{t_{1}-t_{0}}^{x_{0}, y_{0}} \cdots P_{t_{m+1}-t_{m}}^{x_{m}, y_{m}} \quad\left(\text { resp. } \quad P_{t_{m+1}-t_{m}}^{x_{m}, y_{m}} \cdots P_{t_{1}-t_{0}}^{x_{0}, y_{0}}\right)
$$

where $t_{0}=0, t_{m+1}=t,\left\{t_{1}<\ldots<t_{m}\right\}$ is the (possibly empty) union of the sets of discontinuity of $f$ and $g$ in $] 0, t[$ and, for $i=$ $0, \cdots, n, x_{i}:=f\left(t_{i}\right)$ and $y_{i}=g\left(t_{i}\right)$.

(iv) For all $f \in \mathbb{S}_{\mathrm{T}}, g \in \mathbb{S}_{\mathrm{T}^{\prime}}$ and $t \geqslant 0, X_{0}^{f, g}=I$ and, whenever $\{0=$ $\left.s_{0} \leqslant s_{1} \leqslant \ldots \leqslant s_{n+1}=t\right\}$ contains all the points of discontinuity of $f_{[0, t[}$ and $g_{[0, t[}$,

$$
X_{t}^{f, g}=P_{s_{1}-s_{0}}^{x_{0}, y_{0}} \cdots P_{s_{n+1}-s_{n}}^{x_{n}, y_{n}} \quad\left(\text { resp. } \quad P_{s_{n+1}-s_{n}}^{x_{n}, y_{n}} \cdots P_{s_{1}-s_{0}}^{x_{0}, y_{0}}\right)
$$

where, for $j=0, \cdots, n, x_{j}:=f\left(s_{j}\right)$ and $y_{j}:=g\left(s_{j}\right)$.

The adjoint operation on bounded QS processes on a Hilbert space exchanges left and right QS cocycles. As an immediate corollary of the above semigroup decomposition/characterisation it follow that the time-reversal procedure defined in (3.3) does too.

Corollary 4.7. Let $X$ be a bounded $Q S$ process on $\mathfrak{h}$. Then $X$ is a left (respectively right) $Q S$ cocycle if and only if the time-reversed process $X^{R}$ is a right (resp. left) cocycle.

Remark. For a bounded left (respectively, right) QS cocycle $X$ on $\mathfrak{h}$,

$$
\widetilde{X}:=\left(X^{R}\right)^{*}=\left(X^{*}\right)^{R}
$$

defines another bounded left (resp. right) QS cocycle on $\mathfrak{h}$, known as the dual cocycle ([Jou]). 
Cocycle dichotomies. We next give some dichotomies which the cocycle laws entail.

Proposition 4.8. Let $k$ be a $Q S$ cocycle on $\mathrm{V}$, let $j$ be a $Q S$ cocycle on an operator system $\mathrm{W}$, and let $X$ be a (left or right, bounded) operator $Q S$ cocycle on $\mathfrak{h}$. Then each of the following sets is either empty or all of ] $0, \infty[:$

(a) $\left\{t>0: k_{t}\right.$ is injective $\}$;

(b) $\left\{t>0: X_{t}\right.$ is injective $\}$;

(c) assuming that $k$ is completely contractive, $\left\{t>0: k_{t}\right.$ is completely isometric $\}$;

(d) assuming that $j$ is completely positive and contractive, $\left\{t>0: j_{t}\right.$ is unital $\}$

(e) assuming that $X$ is a contraction cocycle,

(i) $\left\{t>0: X_{t}\right.$ is isometric $\}$;

(ii) $\left\{t>0: X_{t}\right.$ is coisometric $\}$.

Proof. For $0 \leqslant r \leqslant t \leqslant u$ where $t>0$, and $l=k$ or $j$,

$$
\begin{aligned}
l_{t} & =\widehat{l}_{t-r} \circ \sigma_{t-r} \circ l_{r} \\
& =\widehat{l}_{r} \circ \sigma_{r} \circ l_{t-r}, \text { and } \\
l_{u} & =\left(\widehat{l}_{t} \circ \sigma_{t}\right)^{\circ N} \circ l_{u-N t},
\end{aligned}
$$

where $N:=\left[t^{-1} u\right]$, so that $0 \leqslant(u-N t) \leqslant t$. Let $t>0$. If $k_{t}$ is injective then (4.12) implies that $k_{r}$ is injective for all $r \leqslant t$. If $k$ is CC and $k_{t}$ is CI then (4.12) implies that

$$
\|A\|=\left\|k_{t}^{(n)}(A)\right\| \leqslant\left\|k_{r}^{(n)}(A)\right\| \leqslant\|A\| \quad \text { for all } n \in \mathbb{N}, A \in \mathrm{M}_{n}(\mathrm{~V}), r \leqslant t
$$

so $k_{r}$ is CI for all $r \leqslant t$. If $j_{t}$ is unital then (4.13) implies that

$$
I=j_{t}(I) \leqslant \widehat{\jmath}_{r}(I) \leqslant I \quad \text { for all } r \leqslant t
$$

so $j_{r}$ is unital for all $r \leqslant t$. Since injectivity or complete isometry for $k_{t}$ (respectively, unitality for $j_{t}$ ) implies the same property for $\left(\widehat{k}_{t} \circ \sigma_{t}\right)$ (resp. $\left.\left(\widehat{\jmath}_{t} \circ \sigma_{t}\right)\right)$, parts (a), (c) and (d) now follow from (4.14).

Since $X$ is injective/isometric/coisometric if and only if the time-reversed process $X^{R}$ is, and $X$ is isometric (respectively coisometric) if and only if the adjoint process $X^{*}$ is coisometric (resp. isometric) it suffices (by Corollary 4.7) to prove (b) and (e ii) in the case that $X$ is a left cocycle. That (b) holds follows by a similar argument to parts (a), (c) and (d), noting that for any $R \in B(\mathfrak{h} \otimes \mathcal{F})$,

$$
\left\{t \geqslant 0: \sigma_{t}(R) \text { is injective }\right\}=\emptyset \text { or } \mathbb{R}_{+} .
$$

Finally, for (eii), let $j_{t}(B):=X_{t}\left(B \otimes I_{\mathcal{F}}\right) X_{t}^{*}$, a CPC cocycle on $B(\mathfrak{h})$ by Proposition 4.5, so that the result follows from (d) since $X$ is coisometric if and only if $j$ is unital.

Associated $\Gamma$-cocycle and global $\Gamma$-semigroup. The associated semigroups $\mathcal{P}^{x, y}$ and $P^{x, y}$ were studied individually in $\left[\mathrm{LW}_{2}\right]$, and when they have bounded generators they can be used to construct a stochastic generator for the cocycle. Here, following Accardi and Kozyrev, we show that the entire family can usefully be treated as a single object. 
Recall the Fock-Weyl operators defined in (3.1). Let $\Gamma$ be a map $I \rightarrow \mathrm{k}$, for some (index) set $I$. Then, writing $\left(\delta^{\alpha}\right)_{\alpha \in I}$ for the usual basis for $l^{2}(I)$,

$$
W_{t}^{\Gamma}: \delta^{\alpha} \otimes \xi \mapsto \delta^{\alpha} \otimes W\left(\Gamma(\alpha)_{[0, t]}\right) \xi, \quad \alpha \in I, \xi \in \mathcal{F},
$$

determines a unitary QS process $W^{\Gamma}$ on $l^{2}(I)$. If $B\left(l^{2}(I) \otimes \mathcal{F}\right)$ is identified with $\mathrm{M}_{I}(B(\mathcal{F}))_{\mathrm{b}}$ then

$$
W_{t}^{\Gamma}=\left[\delta_{\beta}^{\alpha} W\left(\Gamma(\alpha)_{[0, t}[)\right] \in \mathrm{D}_{I}(B(\mathcal{F}))_{\mathrm{b}} .\right.
$$

We consider such processes in three guises. For a total subset $\mathrm{T}$ of $\mathrm{k}$ containing 0 , set $W^{\top}=W^{\Gamma}$ where $\Gamma$ is the inclusion map $\mathrm{T} \rightarrow \mathrm{k}$. For an orthonormal basis $\left(d_{i}\right)_{i \in I_{0}}$ for $\mathrm{k}$, letting

$$
d_{0}=0 \text { in } \mathrm{k}, \quad e_{0}=\left(\begin{array}{l}
1 \\
0
\end{array}\right) \text { and } e_{i}=\left(\begin{array}{c}
0 \\
d_{i}
\end{array}\right) \text { in } \widehat{\mathrm{k}}, \quad \text { and } I=I_{0} \cup\{0\},
$$

so that $\eta=\left(e_{\alpha}\right)_{\alpha \in I}$ is an orthonormal basis for $\widehat{\mathrm{k}}$, set $W^{\eta}=W^{\Gamma}$ where $\Gamma$ is the map $I \rightarrow \mathrm{k}, \alpha \rightarrow d_{\alpha}$. Finally, for $n \geqslant 1$ and $\mathbf{x} \in \mathrm{T}^{n}$, set $W^{\mathbf{x}}=W^{\Gamma}$ where $\Gamma$ is the map $\{1, \ldots, n\} \rightarrow \mathrm{k}, i \mapsto x^{i}$. Thus $W^{\mathrm{T}}$ is a process on $l^{2}(\mathrm{~T})$; $W^{\eta}$ is a process on $\widehat{\mathrm{k}} \cong l^{2}(I)$ consisting of $\eta$-diagonal operators:

$$
W_{t}^{\eta} \in \mathrm{D}_{\eta}(\widehat{\mathrm{k}})_{\mathrm{b} \mathrm{M}} \otimes B(\mathcal{F})
$$

and $W^{\mathbf{x}}$ is the process on $\mathbb{C}^{n}$ given by

$$
W_{t}^{\mathbf{x}}:=\left[\begin{array}{lll}
W\left(x_{[0, t]}^{1}\right) & & \\
& \ddots & \\
& & W\left(x_{[0, t[}^{n}\right)
\end{array}\right] \in \mathrm{M}_{n}(\mathbb{C}) \otimes B(\mathcal{F}) .
$$

The proof of the following is straightforward.

Proposition 4.9. Let $W^{\Gamma}$ be the unitary QS process on $l^{2}(I)$ associated with a map $\Gamma: I \rightarrow \mathrm{k}$, as in (4.15), and let $k$ be a $Q S$ cocycle on an operator space $\mathrm{V}$ with associated semigroups $\left\{\mathcal{P}^{x, y}: x, y \in \mathrm{k}\right\}$.

(a) $W^{\Gamma}$ is a strongly continuous left $Q S$ cocycle.

(b) $W^{\Gamma}$ is also a right $Q S$ cocycle on $l^{2}(I)$.

(c) Each of the associated semigroups of the cocycle $W^{\Gamma}$ is norm continuous if and only if the function $\Gamma$ is bounded.

(d) The completely bounded $Q S$ process on $\mathrm{V} \otimes_{\mathrm{M}} B\left(l^{2}(I)\right)=\mathrm{M}_{I}(\mathrm{~V})_{\mathrm{b}}$ defined by

$$
K_{t}^{\Gamma}:=\left(I_{\mathfrak{h}^{\prime}} \otimes W_{t}^{\Gamma}\right)^{*} k_{t}^{l^{2}(I)}(\cdot)\left(I_{\mathfrak{h}} \otimes W_{t}^{\Gamma}\right)
$$

is a QS cocycle whose expectation semigroup is the Schur-action semigroup given by

$$
\mathcal{P}_{t}^{\Gamma}:=\left[\mathcal{P}_{t}^{\Gamma(\alpha), \Gamma(\beta)}\right]_{\alpha, \beta \in I} .
$$

Remarks. (i) Since the cocycle $W^{\Gamma}$ is unitary, and thus contractive, norm continuity of every associated semigroup follows from norm continuity of its expectation semigroup $\left(\left[\mathrm{LW}_{2}\right]\right)$.

(ii) The proposition applies to $W^{\top}$ for a subset $\mathrm{T}$ of $\mathrm{k}$, or $W^{\eta}$ for an orthonormal basis $\eta$ of $\widehat{k}$ associated with some basis of $k$ as in (4.16). Thus for $\eta=\left(e_{\alpha}\right)_{\alpha \in I}, K^{\eta}$ defines a QS cocycle on $\mathrm{V} \otimes_{\mathrm{M}} B(\widehat{\mathrm{k}})$ whose associated 
expectation semigroup $\mathcal{P}^{\eta}$ is the semigroup of $\eta$-decomposable maps with component semigroups $\mathcal{P}^{(\alpha, \beta)}:=\mathcal{P}^{d_{\alpha}, d_{\beta}}, \alpha, \beta \in I$.

(iii) In part (d) we may take a second function $\Gamma^{\prime}: I^{\prime} \rightarrow \mathrm{k}$ and, by using a rectangular lifting of $k$, obtain a completely bounded QS cocycle on the operator space $\mathrm{V} \otimes_{\mathrm{M}} B\left(l^{2}\left(I^{\prime}\right) ; l^{2}(I)\right)=\mathrm{M}_{I, I^{\prime}}(\mathrm{V})_{\mathrm{b}}$, with Schur-action expectation semigroup

$$
\mathcal{P}^{\Gamma, \Gamma^{\prime}}:=\left(\left[\mathcal{P}_{t}^{\Gamma(\alpha), \Gamma^{\prime}\left(\alpha^{\prime}\right)}\right]_{\alpha \in I, \alpha^{\prime} \in \Gamma^{\prime}}\right)_{t \geqslant 0} .
$$

The semigroups in the collection $\left\{\mathcal{P}^{\mathbf{x}}: \mathbf{x} \in \bigcup_{n \in \mathbb{N}} T^{n}\right\}$ appearing above and in the following section are also seen to be the vacuum expectation semigroups of QS cocycles associated to $k$ through (4.18) and (4.19).

\section{Characterisation and Reconstruction}

In this section we focus our attention initially on completely positive contraction cocycles $k$ on an operator system. We derive additional properties satisfied by the family of associated semigroups, and, following Accardi and Kozyrev, show that conversely, for any such family of semigroups on an operator system indexed by a total subset $\mathrm{T}$ of $\mathrm{k}$ containing 0 , there is a cocycle $k$ for which this is its family of associated semigroups. We then apply Paulsen's $2 \times 2$ matrix trick to extend this characterisation of completely positive contraction cocycles on an operator system to completely contractive cocycles on any operator space. Finally a characterisation of contraction operator cocycles is obtained. Along the way we derive characterisations of completely positive contraction cocycles on a $C^{*}$-algebra and positive contraction operator cocycles on a Hilbert space.

We will repeatedly make use of Schur products beyond the context of scalar matrices (as used in the previous section), but only in the favourable circumstances guaranteed by the following elementary observation.

Lemma 5.1. Let $\mathrm{V}$ be an operator system, or $C^{*}$-algebra, in $B(\mathrm{~h})$, and let $A \in \mathrm{M}_{n}(\mathrm{~V})_{+}$and $\lambda \in \mathrm{M}_{n}(\mathbb{C})_{+}$for some $n \in \mathbb{N}$. Then $A \cdot \lambda:=\left[a_{j}^{i} \lambda_{j}^{i}\right] \in$ $\mathrm{M}_{n}(\mathrm{~V})_{+}$.

Proof. Let $\mathbf{u}=\left(u^{i}\right) \in \mathrm{h}^{n}$. Then, setting $\mu=\lambda^{1 / 2}$ and $\mathbf{u}_{(k)}=\left(\mu_{i}^{k} u^{i}\right)_{i=1}^{n} \in \mathrm{h}^{n}$ $(k=1, \ldots, n)$, we have

$$
\langle\mathbf{u},(A \cdot \lambda) \mathbf{u}\rangle=\sum_{k}\left\langle\mathbf{u}_{(k)}, A \mathbf{u}_{(k)}\right\rangle \geqslant 0 .
$$

Remarks. (i) Using Schur isometries, a version of this result for infinite matrices is exploited in $\left[\mathrm{LW}_{5}\right]$.

(ii) For a commutative $C^{*}$-algebra $\mathrm{A}$, the Schur product of positive elements of $\mathrm{M}_{n}(\mathrm{~A})_{+}$is easily seen to be positive by identifying $\mathrm{M}_{n}(\mathrm{~A})_{+}$with $C\left(\Sigma ; \mathrm{M}_{n}(\mathbb{C})_{+}\right)$, where $\Sigma$ is the spectrum of $\mathrm{A}$.

Completely positive contraction cocycles. Recall the notations $\varpi_{t}^{\mathbf{x}}$ and $\square_{n}$ introduced in (4.7) and (4.9).

Proposition 5.2. Let $k$ be a $Q S$ cocycle on an operator space $\mathrm{V}$, let $\mathrm{T}$ be a total subset of $\mathrm{k}$ containing 0 , and consider the family of semigroups $\left\{\mathcal{P}^{\mathrm{x}}: \mathrm{x} \in \bigcup_{n \geqslant 1} \mathrm{~T}^{n}\right\}$ defined from the associated semigroups of $k$. 
(a) If $\mathrm{V}$ is an operator system, or $C^{*}$-algebra, then the following are equivalent:

(i) The cocycle $k$ is completely positive.

(ii) Each semigroup $\mathcal{P}^{\mathbf{x}}$ is completely positive.

(iii) Each semigroup $\mathcal{P}^{\mathbf{x}}$ is positive.

(b) If $\mathrm{V}$ is an operator system and $k$ is completely positive then the following are equivalent:

(i) $k$ is a contraction cocycle.

(ii) $\mathcal{P}_{t}^{\mathbf{x}}\left(I_{\mathfrak{h}} \otimes \lambda\right) \leqslant I_{\mathfrak{h}} \otimes\left(\varpi_{t}^{\mathbf{x}} \cdot \lambda\right)$ for all $n \geqslant 1, \mathbf{x} \in \mathrm{T}^{n}, \lambda \in \mathrm{M}_{n}(\mathbb{C})_{+}$ and $t \geqslant 0$.

(iii) $\mathcal{P}_{t}^{\mathbf{x}}\left(I_{\mathfrak{h}} \otimes \square_{n}\right) \leqslant I_{\mathfrak{h}} \otimes \varpi_{t}^{\mathbf{x}}$ for all $n \geqslant 1, \mathbf{x} \in \mathrm{T}^{n}$ and $t \geqslant 0$.

Moreover $k$ is unital if and only if equality holds in (ii) (resp. (iii)).

(c) If $\mathrm{V}$ is an operator system and $k$ is completely positive and contractive, then the following are equivalent:

(i) $k$ is unital.

(ii) Equality holds in (b) (ii).

(iii) Equality holds in (b) (iii).

(iv) $\mathcal{P}^{x, x}$ is unital for all $x \in \mathrm{T}$.

Proof. Suppose that $\mathrm{V}$ acts on $\mathfrak{h}$. We start with three observations.

First note that, for $N \geqslant 1, u^{1}, \ldots, u^{N} \in \mathfrak{h}$ and $f_{1}, \ldots, f_{N} \in \mathbb{S}_{\top}^{t}$,

$$
\left\|\sum_{i=1}^{N} u^{i} \otimes \varpi\left(f_{i}\right)\right\|^{2}=\left\langle\mathbf{u},\left[I_{\mathfrak{h}} \otimes\left(\varpi_{t_{1}-t_{0}}^{\mathbf{x}(0)} \cdot \ldots \cdot \varpi_{t_{n+1}-t_{n}}^{\mathbf{x}(n)}\right)\right] \mathbf{u}\right\rangle,
$$

where $\mathbf{x}(k):=\mathbf{f}\left(t_{k}\right) \in \mathrm{T}^{N}$ for $k=1, \ldots, n$, whenever $\left\{0=t_{0} \leqslant \cdots \leqslant t_{n}=t\right\}$ contains the discontinuities of each $f_{i}$.

Secondly,

$$
\sum_{i, j=1}^{N}\left\langle u^{i} \otimes \varpi\left(f_{i}\right), k_{t}\left(a_{j}^{i}\right) u^{j} \otimes \varpi\left(f_{j}\right)\right\rangle=\left\langle\mathbf{u}, k_{t}^{\mathbf{f}}\left(\left[a_{j}^{i}\right]\right) \mathbf{u}\right\rangle
$$

where $k^{\mathbf{f}}$ is the Schur-action map on $\mathrm{M}_{N}(\mathrm{~V})$ from (4.4).

Thirdly, the isometries defined by $F_{\mathbf{x}, t}:=\left(I_{\mathfrak{h}} \otimes W_{t}^{\mathbf{x}}\right) E(0), \mathbf{x} \in \mathrm{T}^{n}$ (in the notation (3.2)), satisfy

$$
F_{\mathbf{x}, t}^{*}\left(I_{\mathfrak{h}} \otimes \lambda \otimes I_{\mathcal{F}}\right) F_{\mathbf{x}, t}=I_{\mathfrak{h}} \otimes\left(\varpi_{t}^{\mathbf{x}} \cdot \lambda\right)
$$

for each $\lambda \in \mathrm{M}_{n}(\mathbb{C})$.

(a) If $k$ is $\mathrm{CP}$ then each $\mathcal{P}^{\mathrm{x}}$ is $\mathrm{CP}$ by Proposition $4.9(\mathrm{~d})$. Conversely, suppose that each $\mathcal{P}^{\mathbf{x}}$ is positive. Then, by a reindexing, we see that each $\left(\mathcal{P}_{t}^{\mathbf{x}}\right)^{(n)}$ is of the form $\mathcal{P}_{t}^{\mathbf{x}^{\prime}}$, and so each $\mathcal{P}^{\mathbf{x}}$ is actually CP and therefore, by Proposition 4.3, each $k_{t}^{\mathbf{f}}$ is CP. By adaptedness, the density of $\mathfrak{h} \otimes \mathcal{E}_{\mathrm{T}}^{t}$ in $\mathfrak{h} \otimes \mathcal{F}^{t}$ and (4.8), it follows from (5.2) that $k$ is CP.

(b) Suppose that the conditions of (a) hold and let $\Sigma$ be the tensor flip $\mathrm{V} \otimes_{\mathrm{M}} B\left(\mathcal{F} \otimes \mathbb{C}^{n}\right) \rightarrow \mathrm{V} \otimes_{\mathrm{M}} B\left(\mathbb{C}^{n} \otimes \mathcal{F}\right)$. If $k$ is also contractive then, by Proposition 4.9(d),

$$
\mathcal{P}_{t}^{\mathbf{x}}\left(I_{\mathfrak{h}} \otimes \lambda\right)=F_{\mathbf{x}, t}^{*} \Sigma\left(k_{t}\left(I_{\mathfrak{h}}\right) \otimes \lambda\right) F_{\mathbf{x}, t} \leqslant F_{\mathbf{x}, t}^{*}\left(I_{\mathfrak{h}} \otimes \lambda \otimes I_{\mathcal{F}}\right) F_{\mathbf{x}, t}
$$

for $\lambda \in \mathrm{M}_{n}(\mathbb{C})_{+}$, so (ii) holds by (5.3). 
If (iii) holds then for any $\lambda \in \mathrm{M}_{n}(\mathbb{C})_{+}$

$$
\mathcal{P}_{t}^{\mathbf{x}}\left(I_{\mathfrak{h}} \otimes \lambda\right)=\mathcal{P}_{t}^{\mathbf{x}}\left(I_{\mathfrak{h}} \otimes \square_{n}\right) \cdot \lambda \leqslant I_{\mathfrak{h}} \otimes\left(\varpi_{t}^{\mathbf{x}} \cdot \lambda\right)
$$

by Lemma 5.1. Thus (ii) holds.

Now suppose that (ii) holds and let $\xi=\sum_{i=1}^{N} u^{i} \otimes \varpi\left(f_{i}\right)$ where $\mathbf{f} \in\left(\mathbb{S}_{\mathrm{T}}^{t}\right)^{n}$ has all its discontinuities in $\left\{0=t_{0} \leqslant \cdots \leqslant t_{n+1}=t\right\}$. Then, by (5.2), (4.4) and (5.1)

$$
\begin{aligned}
\left\langle\xi, k_{t}\left(I_{\mathfrak{h}}\right) \xi\right\rangle & =\left\langle\mathbf{u}, k_{t}^{\mathbf{f}}\left(I_{\mathfrak{h}} \otimes \square_{N}\right) \mathbf{u}\right\rangle \\
& \leqslant\left\langle\mathbf{u},\left[I_{\mathfrak{h}} \otimes\left(\varpi_{t_{1}-t_{0}}^{\mathbf{x}(0)} \cdots \varpi_{t_{n+1}-t_{n}}\right)\right] \mathbf{u}\right\rangle=\|\xi\|^{2} .
\end{aligned}
$$

Thus, by complete positivity, $k$ is a contraction cocycle.

Since (iii) is a special case of (ii), this proves the equivalences.

(c) Tracing back through the proof of (b) confirms the equivalence of (i), (ii) and (iii). In case $n=1$, (iii) reads $\mathcal{P}_{t}^{x, x}\left(I_{\mathfrak{h}}\right)=I_{\mathfrak{h}}$ for all $x \in \mathrm{T}$ and $t \in \mathbb{R}_{+}$, so (iii) implies (iv). Conversely, since $k$ is assumed CP and contractive, we have from (b) (iii) that

$$
0 \leqslant I_{\mathfrak{h}} \otimes \varpi_{t}^{\mathbf{x}}-\mathcal{P}_{t}^{\mathbf{x}}\left(I_{\mathfrak{h}} \otimes \square_{n}\right)
$$

for each $n \geqslant 1, \mathbf{x} \in \mathrm{T}^{n}$ and $t \geqslant 0$, so if (iv) holds then the entries on the diagonal in the difference above are all 0 . Therefore (iii) holds (by the remark following (1.3)).

If $f_{1}, \ldots, f_{n}$ are distinct vectors in a Hilbert space, $u_{1}, \ldots, u_{n}$ are any vectors from another Hilbert space and $\sum u_{i} \otimes \varpi\left(f_{i}\right)=0$, then $u_{1}=\cdots=$ $u_{n}=0$. This straightforward extension of the well-known linear independence of exponential vectors implies the following result, in which we adopt the notations

$$
\mathcal{E}_{\mathrm{T}, t}:=\operatorname{Lin}\left\{\varpi(f): f \in \mathbb{S}_{\mathrm{T}, t}\right\} \text { where } \mathbb{S}_{\mathrm{T}, t}:=\left\{f \in \mathbb{S}_{\mathrm{T}}: \operatorname{supp} f \subset[0, t[\} .\right.
$$

Lemma 5.3. For a Hilbert space $\mathfrak{h}$, a subset $\mathrm{T}$ of $\mathrm{k}$, a vector space $U$ and a function $\psi:\left(\mathfrak{h} \times \mathbb{S}_{\mathrm{T}, t}\right) \times\left(\mathfrak{h} \times \mathbb{S}_{\mathrm{T}, t}\right) \rightarrow U$, if each function $(u, v) \mapsto$ $\psi((u, f),(v, g))$ is sesquilinear $\mathfrak{h} \times \mathfrak{h} \rightarrow U$ then there is a unique sesquilinear map $\Psi:\left(\mathfrak{h} \otimes \mathcal{E}_{\mathrm{T}, t}\right) \times\left(\mathfrak{h} \otimes \mathcal{E}_{\mathrm{T}, t}\right) \rightarrow U$ satisfying

$$
\Psi(u \otimes \varpi(f), v \otimes \varpi(g))=\psi((u, f),(v, g)) .
$$

We now begin the task of reconstruction of cocycles from classes of semigroups.

Theorem 5.4. Let $\mathrm{V}$ be an operator system and let $\mathcal{S}:=\left\{\mathcal{P}^{x, y}: x, y \in \mathrm{T}\right\}$ be a family of semigroups on $\mathrm{V}$ indexed by a total subset $\mathrm{T}$ of $\mathrm{k}$ containing 0 . Suppose that $\mathcal{S}$ satisfies the following conditions:

(a) Each semigroup $\mathcal{P}^{\mathbf{x}}$ is positive, and

(b) $\mathcal{P}_{t}^{\mathbf{x}}\left(I_{\mathfrak{h}} \otimes \square_{n}\right) \leqslant I_{\mathfrak{h}} \otimes \varpi_{t}^{\mathbf{x}}$ for all $n \geqslant 1, \mathbf{x} \in \mathrm{T}^{n}$ and $t \geqslant 0$.

Then there is a unique completely positive contraction cocycle $k$ on $\mathrm{V}$ whose associated semigroups include $\mathcal{S}$. Moreover, $k$ is unital if and only if each inequality in (b) is an equality. 
Proof. In this proof we identify elements of $\mathbb{S}_{\mathrm{T}, t}$ with their restrictions to $[0, t[$.

Suppose that $\vee$ acts on $\mathfrak{h}$. First note that the proof ( $b$ iii $\Rightarrow b$ ii) in Proposition 5.2 applies to $\mathcal{S}$, thus we may assume that this family satisfies

$(\mathrm{b})^{\prime} \mathcal{P}_{t}^{\mathbf{x}}\left(I_{\mathfrak{h}} \otimes \lambda\right) \leqslant I_{\mathfrak{h}} \otimes\left(\varpi_{t}^{\mathbf{x}} \cdot \lambda\right)$ for all $n \geqslant 1, \mathbf{x} \in \mathrm{T}^{n}, \lambda \in \mathrm{M}_{n}(\mathbb{C})_{+}$and $t \geqslant 0$.

For each $t>0$ and $f, g \in \mathbb{S}_{\mathrm{T}, t}$ define a bounded map $k(f, g, t)$ on $\mathrm{V}$ by

$$
k(f, g, t)=\mathcal{P}_{t_{1}-t_{0}}^{x_{0}, y_{0}} \circ \cdots \circ \mathcal{P}_{t_{n+1}-t_{n}}^{x_{n}, y_{n}}
$$

where $\left\{0=t_{0} \leqslant \cdots \leqslant t_{n+1}=t\right\}$ contains the discontinuities of $f$ and $g$, and $\left(x_{i}, y_{i}\right)=\left(f\left(t_{i}\right), g\left(t_{i}\right)\right)$. That these maps are well-defined, that is independent of the choice of subdivision of $[0, t[$, is a consequence of the semigroup property of each $\mathcal{P}^{x, y}$.

Now, for each $(u, v) \in \mathfrak{h} \times \mathfrak{h}$, the map

$$
\mathrm{V} \rightarrow \mathbb{C}, \quad a \mapsto\langle u, k(f, g, t)(a) v\rangle
$$

defines a bounded linear functional on $\mathrm{V}$. Thus Lemma 5.3 implies the existence of a sesquilinear map $\left(\mathfrak{h} \underline{\otimes} \mathcal{E}_{\mathrm{T}, t}\right) \times\left(\mathfrak{h} \underline{\otimes} \mathcal{E}_{\mathrm{T}, t}\right) \rightarrow \mathrm{V}^{*}$, denoted $(\xi, \eta) \mapsto$ $k_{t}[\xi, \eta]$, satisfying

$$
k_{t}[\xi, \eta](a)=\sum_{i, j=1}^{N}\left\langle u^{i}, k\left(f_{i}, g_{j}, t\right)(a) v^{j}\right\rangle
$$

for $\xi=\sum_{i=1}^{N} u^{i} \otimes \varpi\left(f_{i}\right)$ and $\eta=\sum_{j=1}^{N} v^{j} \otimes \varpi\left(g_{j}\right)$ in $\underline{h} \otimes \mathcal{E}_{\mathrm{T}, t}$. In particular, if $a \in \mathrm{V}_{+}$then letting $\mathbf{x}(k)=\mathbf{f}\left(t_{k}\right) \in \mathrm{T}^{N}$ for $k=0, \ldots, n$, where $\left\{0=t_{0} \leqslant\right.$ $\left.\cdots \leqslant t_{n+1}=t\right\}$ contains the discontinuities of $\mathbf{f}$, and using the inequality $a \otimes \square_{N} \leqslant\|a\| I_{\mathfrak{h}} \otimes \square_{N},(5.1)$ implies that

$$
\begin{aligned}
k_{t}[\xi, \xi](a) & =\left\langle\mathbf{u}, \mathcal{P}_{t_{1}-t_{0}}^{\mathbf{x}(0)} \circ \cdots \circ \mathcal{P}_{t_{n+1}-t_{n}}^{\mathbf{x}(n)}\left(a \otimes \square_{n}\right) \mathbf{u}\right\rangle \\
& \leqslant\|a\|\left\langle\mathbf{u},\left[I_{\mathfrak{h}} \otimes\left(\varpi_{t_{1}-t_{0}}^{\mathbf{x}(0)} \cdots \varpi_{t_{n+1}-t_{n}}^{\mathbf{x}(n)}\right] \mathbf{u}\right\rangle=\|a\|\|\xi\|^{2} .\right.
\end{aligned}
$$

Since $\mathrm{V}=\operatorname{Lin} \mathrm{V}_{+}$this implies that, for any $a \in \mathrm{V}$, the quadratic form $\xi \mapsto k_{t}[\xi, \xi](a)$ is bounded. Therefore there is a bounded operator $k(t, a)$ on $\mathfrak{h} \otimes \mathcal{F}_{[0, t[}$ satisfying $\langle\xi, k(t, a) \xi\rangle=k_{t}[\xi, \xi](a)$. Moreover, by (5.4),

$$
k(t, a) \geqslant 0 \text { for } a \in \mathrm{V}_{+} .
$$

By the linearity of each $k_{t}[\xi, \xi], k(t, a)$ is linear in $a$ and so $k_{t}(a)=k(t, a) \otimes$ $I_{[t, \infty[}$ defines an adapted family of linear maps $k_{t}: \bigvee \rightarrow B(\mathfrak{h} \otimes \mathcal{F})$. By $(5.5)$ each $k_{t}$ is positive and so also bounded (by Proposition 1.1). Since, for $f, g \in \mathbb{S}_{\mathrm{T}}$,

$$
k_{t}^{f, g}=k\left(\left.f\right|_{[0, t[},\left.g\right|_{[0, t[}, t\right)=\mathcal{P}_{t_{1}-t_{0}}^{x_{0}-y_{0}} \circ \cdots \circ \mathcal{P}_{t_{n+1}-t_{n}}^{x_{n}-y_{n}},
$$

where $\left(x_{l}, y_{l}\right):=\left(f\left(t_{l}\right), g\left(t_{l}\right)\right)$ for $l=1, \ldots, n$ and $\left\{0=t_{0} \leqslant \cdots \leqslant t_{n+1}=\right.$ $t\}$ contains the discontinuities of both $f_{[0, t[}$ and $g_{[0, t[}, k$ is a process on $\mathrm{V}$ (Lemma 2.1); it is therefore a bounded positive QS cocycle on $\mathrm{V}$ whose associated semigroups include $\mathcal{S}$, by Proposition 4.1. Complete positivity and contractivity of $k$ now follow from Proposition 5.2. Uniqueness follows from Corollary 4.2. The last part is contained in Proposition 5.2. 
In $\left[\mathrm{AK}_{2}\right]$ the characterisation of CP unital QS cocycles on $B(\mathfrak{h})$ with onedimensional $\mathrm{k}$ is given in terms of a single Schur-action CP semigroup on $\mathrm{M}_{2}(B(\mathfrak{h}))$, rather than a family $\mathcal{S}=\left\{\mathcal{P}^{x, y}: x, y \in \mathrm{T}\right\}$ of semigroups on $\mathrm{V}$ as above. We next show two ways in which such a global characterisation can be obtained for cocycles on operator systems with multidimensional noise; the first requires a separability assumption. Recall the definition of $\mathcal{P}^{\top}$ for a QS cocycle and total subset $\mathrm{T}$ of $\mathrm{k}$ containing 0 (Proposition 4.9).

Theorem 5.5. Let $\mathrm{k}$ be separable, let $\mathrm{T}$ be a countable total subset of $\mathrm{k}$ that contains 0 and let $\mathcal{P}$ be a semigroup on $\mathrm{M}_{\mathrm{T}}(\mathrm{V})_{\mathrm{b}}$ for an operator system $\mathrm{V}$. Then $\mathcal{P}$ is of the form $\mathcal{P}^{\top}$ for some completely positive contraction cocycle if and only if

(a) $\mathcal{P}$ has Schur-action,

(b) $\mathcal{P}$ is completely positive, and

(c) for some $\zeta=\left(\zeta^{x}\right) \in l^{2}(\mathrm{~T})$ with $\zeta^{x} \neq 0$ for each $x \in \mathrm{T}$ we have

$$
\mathcal{P}_{t}\left(I_{\mathfrak{h}} \otimes \Lambda\right) \leqslant I_{\mathfrak{h}} \otimes\left(\varpi_{t}^{\top} \cdot \Lambda\right) \text { for all } t \geqslant 0
$$

$$
\text { where } \Lambda=|\zeta\rangle\langle\zeta| \in B\left(l^{2}(\mathrm{~T})\right)=\mathrm{M}_{\mathrm{T}}(\mathbb{C})_{\mathrm{b}} \text {. }
$$

In this case (5.6) holds for all $\Lambda \in B\left(l^{2}(\mathrm{~T})\right)_{+}$.

Remark. The statement above already illustrates one issue arising with the passage to multidimensions: in general $\varpi_{t}^{\top}:=\left[\left\langle\varpi\left(x_{[0, t]}\right), \varpi\left(y_{[0, t}\right)\right\rangle\right]_{x, y \in \mathbf{T}}$ will not define an element of $B\left(l^{2}(\mathrm{~T})\right)$. However, it is a Schur multiplier, with the map $\Lambda \mapsto \varpi_{t}^{\top} \cdot \Lambda$ being $\mathrm{CP}$ and unital on $B\left(l^{2}(\mathbf{T})\right)$; indeed

$$
\varpi_{t}^{\top} \cdot \Lambda:=F_{\mathrm{T}, t}^{*}\left(I_{\mathfrak{h}} \otimes \Lambda \otimes I_{\mathcal{F}}\right) F_{\mathrm{T}, t}
$$

where $F_{\mathrm{T}, t}:=\left(I_{\mathfrak{h}} \otimes W_{t}^{\top}\right) E(0)$ (cf. the earlier $F_{\mathbf{x}, t}$ notation).

Proof. That (5.6) holds when $\mathcal{P}$ is of the form $\mathcal{P}^{\top}$, for some completely positive contraction cocycle, follows by the same argument as in the proof of Proposition 5.2. Assume, conversely, that $\mathcal{P}$ has Schur-action and (5.6) holds for $\Lambda$ of the given form. Then $\mathcal{P}$ has components $\left[\mathcal{P}_{t}^{x, y}\right]_{x, y \in \mathrm{T}}$ as defined through (2.5). Let $n \geqslant 1$ and $\mathbf{x} \in \mathrm{T}^{n}$, set $\mathcal{P}_{t}^{\mathbf{x}}=\left[\mathcal{P}_{t}^{x_{i}, x_{j}}\right]: \mathrm{M}_{n}(\mathrm{~V}) \rightarrow \mathrm{M}_{n}(\mathrm{~V})$, and note that

$$
\mathcal{P}_{t}^{\mathbf{x}}(A)=G_{\mathbf{x}}^{*} \mathcal{P}_{t}^{(n)}\left(G_{\mathbf{x}} A G_{\mathbf{x}}^{*}\right) G_{\mathbf{x}}
$$

where $G_{\mathbf{x}}=\operatorname{diag}\left[G_{1} \cdots G_{n}\right]: \mathfrak{h}^{n} \rightarrow\left(\mathfrak{h} \otimes l^{2}(\mathbf{T})\right)^{n}$ is the diagonal operator with $G_{i}=E_{y}$ for $y=\delta^{x_{i}}$. Thus $\mathcal{P}_{t}^{\mathbf{x}}$ is a positive map. Moreover, setting $\lambda^{\mathbf{x}}:=\left(\zeta^{x_{i}}\right) \in \mathbb{C}^{n}$

$$
\begin{aligned}
\mathcal{P}_{t}^{\mathbf{x}}\left(I_{\mathfrak{h}} \otimes \square_{n}\right) \cdot\left|\lambda^{\mathbf{x}}\right\rangle\left\langle\lambda^{\mathbf{x}}\right| & =\mathcal{P}_{t}^{\mathbf{x}}\left(I_{\mathfrak{h}} \otimes\left|\lambda^{\mathbf{x}}\right\rangle\left\langle\lambda^{\mathbf{x}}\right|\right) \\
& =G_{\mathbf{x}}^{*}\left(\mathcal{P}_{t}\left(I_{\mathfrak{h}} \otimes|\zeta\rangle\langle\zeta|\right) \otimes \square_{n}\right) G_{\mathbf{x}} \\
& \leqslant G_{\mathbf{x}}^{*}\left(I_{\mathfrak{h}} \otimes\left(\varpi_{t}^{\top} \cdot|\zeta\rangle\langle\zeta|\right) \otimes \square_{n}\right) G_{\mathbf{x}} \\
& =\left(I_{\mathfrak{h}} \otimes \varpi_{t}^{\mathbf{x}}\right) \cdot\left|\lambda^{\mathbf{x}}\right\rangle\left\langle\lambda^{\mathbf{x}}\right| .
\end{aligned}
$$

Setting $\mu^{\mathbf{x}}:=\left(1 / \zeta^{x_{i}}\right) \in \mathbb{C}^{n}$, then $\left|\mu^{\mathbf{x}}\right\rangle\left\langle\mu^{\mathbf{x}}\right| \in B\left(\mathbb{C}^{n}\right)_{+}=\mathrm{M}_{n}(\mathbb{C})_{+}$is the Schur inverse of $\left|\lambda^{\mathbf{x}}\right\rangle\left\langle\lambda^{\mathbf{x}}\right|$, and so

$$
\mathcal{P}_{t}^{\mathbf{x}}\left(I_{\mathfrak{h}} \otimes \square_{n}\right) \leqslant I_{\mathfrak{h}} \otimes \varpi_{t}^{\mathbf{x}}
$$

by Lemma 5.1. Thus Theorem 5.4 applies giving the existence of a cocycle $k$ with global semigroup $\mathcal{P}$. 
Remark. If $\mathrm{T} \subset \mathrm{k}$ is uncountable then, for any $\Lambda \in B\left(l^{2}(\mathrm{~T})\right)=\mathrm{M}_{\mathrm{T}}(\mathbb{C})_{\mathrm{b}}$, only countably many components of $\Lambda$ in each row and column can be nonzero, and therefore many finite submatrices will not be Schur-invertible.

Specialising to the case of unital cocycles we next give conditions that guarantee the Schur-action of the global semigroup.

Theorem 5.6. Let $\mathcal{P}$ be a semigroup on $\mathrm{M}_{\mathrm{T}}(\mathrm{V})_{\mathrm{b}}$ for an operator system $\mathrm{V}$ and a total subset $\mathrm{T}$ of $\mathrm{k}$ containing 0 . Then $\mathcal{P}$ is of the form $\mathcal{P}^{\top}$ for some completely positive unital $Q S$ cocycle on $\mathrm{V}$ if and only if $\mathcal{P}$ is completely positive, contractive and satisfies the normalisation conditions

$$
\mathcal{P}_{t}\left(I_{\mathfrak{h}} \otimes\left|\delta^{x}\right\rangle\left\langle\delta^{y}\right|\right)=e^{-t \chi(x, y)} I_{\mathfrak{h}} \otimes\left|\delta^{x}\right\rangle\left\langle\delta^{y}\right|, \quad x, y \in \mathrm{T}, t \geqslant 0 .
$$

Proof. If $\mathcal{P}=\mathcal{P}^{\top}$ for a $\mathrm{CP}$ unital $\mathrm{QS}$ cocycle on $\mathrm{V}$ then it is clear from the definition that $\mathcal{P}$ is $\mathrm{CP}$ and satisfies

$$
\mathcal{P}_{t}\left(I_{\mathfrak{h}} \otimes\left|\delta^{x}\right\rangle\left\langle\delta^{y}\right|\right)=\left\langle\varpi\left(x_{[0, t[}\right), \varpi\left(y_{[0, t]}\right)\right\rangle I_{\mathfrak{h}} \otimes\left|\delta^{x}\right\rangle\left\langle\delta^{y}\right|,
$$

which equals the right hand side of (5.7).

Suppose conversely that $\mathcal{P}$ is a $\mathrm{CP}$ semigroup on $\mathrm{M}_{\mathrm{T}}(\mathrm{V})$ satisfying (5.7). Then $\mathcal{P}_{t}\left(I_{\mathfrak{h}} \otimes\left|\delta^{x}\right\rangle\left\langle\delta^{x}\right|\right)=I_{\mathfrak{h}} \otimes\left|\delta^{x}\right\rangle\left\langle\delta^{x}\right|$ for each $x \in \mathrm{T}$ and so, by Proposition 2.6, $\mathcal{P}_{t}$ has Schur-action. Positivity of each $\mathcal{P}^{\mathrm{x}}$ follows by a standard reindexing argument, and each $\mathcal{P}^{\mathbf{x}}$ is easily seen to satisfy the conditions of Theorem 5.4(b) with equality, so the proof is complete.

Remark. The single normalisation condition cited in Theorem 21 of $\left[\mathrm{AK}_{2}\right]$ is not sufficient to guarantee that the semigroup $\mathcal{P}$ has Schur-action. For example, let $\mathfrak{h}=\mathbf{k}=\mathbb{C}$, so that $\mathbf{V}=\mathbb{C}$, and let $\mathbf{T}=\{0,1\}$. Let $\Phi$ be the completely positive map on $\mathrm{M}_{2}(\mathbb{C})$ defined by $\Phi\left(\left[\begin{array}{ll}a & b \\ c & d\end{array}\right]\right)=\left[\begin{array}{ll}d & 0 \\ 0 & a\end{array}\right]$, and define $\Psi$ by $\Psi(A)=\Phi(A)-A$. Then $\Psi$ is the generator of a unital completely positive semigroup $\mathcal{P}$ on $\mathrm{M}_{2}(\mathbb{C})([\mathrm{GKS}])$ satisfying

$$
\Psi\left(\left[\begin{array}{ll}
a & b \\
c & d
\end{array}\right]\right)=\left[\begin{array}{cc}
d-a & -b \\
-c & a-d
\end{array}\right] \text { and } \mathcal{P}_{t}\left(\left[\begin{array}{ll}
1 & 1 \\
1 & 1
\end{array}\right]\right)=\left[\begin{array}{cc}
1 & e^{-t / 2} \\
e^{-t / 2} & 1
\end{array}\right]
$$

This semigroup satisfies the normalisation condition of $\left[\mathrm{AK}_{2}\right]$ (modified for the use of normalised exponential vectors) but clearly does not have Schuraction and so cannot be the global semigroup of a QS cocycle.

Cocycles on a $C^{*}$-algebra. Let $R$ and $T$ be self-adjoint operators on a Hilbert space $\mathfrak{h}$. Denote the set $\{S \in B(\mathfrak{h}): R \leqslant S \leqslant T\}$ by $[R, T]$, and for a $C^{*}$-algebra $A$ acting on $\mathfrak{h}$ define

$$
[R, T]_{\mathrm{A}}:=[R, T] \cap \mathrm{A} .
$$

Theorem 5.7. Let $\mathrm{C}$ be a nonunital $C^{*}$-algebra acting nondegenerately, let $\mathrm{T}$ be a total subset of $\mathrm{k}$ containing 0 , and let $\mathcal{S}=\left\{\mathcal{P}^{x, y}: x, y \in \mathrm{T}\right\}$ be a family of semigroups on $\mathrm{C}$. Then there is a completely positive contraction cocycle on $\mathrm{C}$ whose associated semigroups include $\mathcal{S}$ if and only if the family $\mathcal{S}$ satisfies

$$
\mathcal{P}_{t}^{\mathbf{x}}\left(\left[0, I_{\mathfrak{h}} \otimes \lambda\right]_{\mathrm{M}_{n}(\mathrm{C})}\right) \subset\left[0, I_{\mathfrak{h}} \otimes\left(\lambda \cdot \varpi_{t}^{\mathbf{x}}\right)\right]_{\mathrm{M}_{n}(\mathrm{C})}
$$

for all $n \geqslant 1, \mathbf{x} \in \mathrm{T}^{n}, \lambda \in \mathrm{M}_{n}(\mathbb{C})_{+}$and $t \geqslant 0$. 
Proof. Let $\mathfrak{h}$ be the Hilbert space on which $\mathrm{C}$ acts.

First let $k$ be a completely positive contraction cocycle on $C$. For each $t$ let $j_{t}$ be the extension of $k_{t}$ to the unitisation of $C$ in $B(\mathfrak{h})$ defined by

$$
j_{t}:{ }^{u} \mathrm{C} \rightarrow B(\mathfrak{h} \otimes \mathcal{F}), \quad a+z I_{\mathfrak{h}} \mapsto k_{t}(a)+z I_{\mathfrak{h} \otimes \mathcal{F}} .
$$

Thus (by Proposition 1.3) $j_{t}$ is $\mathrm{CP}$ and unital, and since

$$
j_{t}^{f, g}\left(a+z I_{\mathfrak{h}}\right)=k_{t}^{f, g}(a)+\left\langle\varpi\left(f_{[0, t[}\right), \varpi\left(g_{[0, t[}\right)\right\rangle z I_{\mathfrak{h} \otimes \mathcal{F}},
$$

it follows from Proposition 4.1 that $j$ is a QS cocycle on ${ }^{u} \mathrm{C}$. Let $\left\{\mathcal{R}^{x, y}\right.$ : $x, y \in \mathrm{k}\}$ be its family of associated semigroups. Then, for $\lambda \in \mathrm{M}_{n}(\mathbb{C})_{+}$, $A \in\left[0, I_{\mathfrak{h}} \otimes \lambda\right]_{\mathrm{M}_{n}(\mathrm{C})}$ and $\mathbf{x} \in \mathrm{T}^{n}$,

$$
0 \leqslant \mathcal{P}_{t}^{\mathbf{x}}(A)=\mathcal{R}_{t}^{\mathbf{x}}(A) \leqslant \mathcal{R}_{t}^{\mathbf{x}}\left(I_{\mathfrak{h}} \otimes \lambda\right)=I_{\mathfrak{h}} \otimes\left(\lambda \cdot \varpi_{t}^{\mathbf{x}}\right)
$$

by Proposition 5.2, as required.

The proof of the converse follows that of Theorem 5.4, except that now the property $k_{t}[\xi, \xi](a) \leqslant\|a\|\|\xi\|^{2}$ follows from a careful iteration of (5.8). Contractivity of the resulting cocycle follows from Proposition 1.3.

Positive contraction operator cocycles. We now apply the results of this section to the case of positive contraction operator cocycles. This class has been studied in [W] under the assumption of Markov regularity - that is, norm-continuity of its expectation semigroup, and in [Bha], under the assumption of locality, or being a pure noise cocycle - that is, $X_{t} \in I_{\mathfrak{h}} \otimes$ $B\left(\mathcal{F}_{[0, t]}\right) \otimes I_{[t, \infty[}$ for all $t \in \mathbb{R}_{+}$.

Theorem 5.8. Let $\mathrm{T}$ be a total subset of $\mathrm{k}$ containing 0 and let $S=\left\{P^{x, y}\right.$ : $x, y \in \mathrm{T}\}$ be a family of semigroups on a Hilbert space $\mathfrak{h}$. Then there is a positive contraction operator cocycle on $\mathfrak{h}$ whose associated semigroups include $S$ if and only if $S$ satisfies the following conditions:

(a) the family of operators $\left\{P_{t}^{x, y}: x, y \in \mathrm{T}, t \geqslant 0\right\}$ is commutative, and

(b) $0 \leqslant P_{t}^{\mathbf{x}} \leqslant I_{\mathfrak{h}} \otimes \varpi_{t}^{\mathbf{x}}$ for all $n \geqslant 1, \mathbf{x} \in \mathrm{T}^{n}$, and $t \geqslant 0$.

Proof. Define $\mathrm{C}:=C^{*}\left\{P_{t}^{x, y}: x, y \in \mathrm{T}, t \geqslant 0\right\}$.

Let $X$ be a positive contraction cocycle on $\mathfrak{h}$ with associated semigroups $\left\{P_{t}^{x, y}: x, y \in \mathrm{k}\right\}$. By self-adjointness $X$ is also a right cocycle and so, by the semigroup decomposition (4.11), it follows that (a) holds. The completely bounded QS process on $\mathrm{C}$ defined by $k_{t}(a)=X_{t}\left(a \otimes I_{\mathcal{F}}\right)=\left(a \otimes I_{\mathcal{F}}\right) X_{t}$ is a completely positive contraction cocycle whose associated semigroups are given by $\mathcal{P}_{t}^{x, y}(a)=P_{t}^{x, y} a$. Therefore $P_{t}^{\mathbf{x}}=\mathcal{P}_{t}^{\mathbf{x}}\left(I_{\mathfrak{h}} \otimes \square_{n}\right)$ for $n \geqslant 1$ and $\mathrm{x} \in \mathrm{k}^{n}$, and so (b) holds by Proposition 5.2.

Conversely, suppose that $S$ satisfies (a) and (b). Then $\mathrm{C}$ is unital and abelian and $\mathcal{P}_{t}^{x, y}(a):=P_{t}^{x, y} a$ defines a family of semigroups $\mathcal{S}=\left\{\mathcal{P}^{x, y}\right.$ : $x, y \in \mathrm{T}\}$ on $\mathrm{C}$ satisfying $\mathcal{P}_{t}^{\mathbf{x}}\left(I_{\mathfrak{h}} \otimes \square_{n}\right) \leqslant I_{\mathfrak{h}} \otimes \varpi_{t}^{\mathbf{x}}$. Since $\mathcal{P}_{t}^{\mathbf{x}}(A)=P_{t}^{\mathbf{x}} \cdot A$, it follows from the remark after Lemma 5.1 that the semigroups $\mathcal{P}^{\mathbf{x}}$ are positive. Thus Theorem 5.4 implies that there is a completely positive contraction cocycle $k$ on $C$ whose associated semigroups include $\mathcal{S}$; the associated semigroups of the positive contraction operator cocycle on $\mathfrak{h}$ defined by $X_{t}=k_{t}\left(I_{\mathfrak{h}}\right)$ therefore include $S$. 
Completely contractive quantum stochastic cocycles. We now apply Paulsen's $2 \times 2$ matrix trick to deal with completely contractive cocycles on operator spaces.

Set

$$
\begin{aligned}
\mathrm{M}_{n}(\mathbb{C})_{++}: & =\left\{\lambda \in \mathrm{M}_{n}(\mathbb{C}): \lambda \text { is uniformly positive }\right\} \\
& =\mathrm{M}_{n}(\mathbb{C})_{+} \cap \mathrm{GL}_{n}(\mathbb{C}) .
\end{aligned}
$$

Lemma 5.9. If $\lambda \in \mathrm{M}_{n}(\mathbb{C})_{++}$then $\lambda \cdot \varpi_{t}^{\mathbf{x}} \in \mathrm{M}_{n}(\mathbb{C})_{++}$for each $\mathbf{x}$ in $\mathrm{k}^{n}$.

Proof. If $\lambda \geqslant c I$ then, since $\varpi_{t}^{\mathbf{x}} \geqslant 0$, we have $\lambda \cdot \varpi_{t}^{\mathbf{x}} \geqslant c I \cdot \varpi_{t}^{\mathbf{x}}=c I$. The result follows.

Theorem 5.10. Let $\mathrm{V}$ be an operator space, let $\mathrm{T}$ be a total subset of $\mathrm{k}$ containing 0 , and let $\mathcal{S}=\left\{\mathcal{P}^{x, y}: x, y \in \mathrm{T}\right\}$ be a family of semigroups on $\mathrm{V}$. Then the following are equivalent:

(i) There is a completely contractive $Q S$ cocycle on $\mathrm{V}$ whose associated semigroups include $\mathcal{S}$.

(ii) Each semigroup $\widetilde{\mathcal{P}}^{\mathbf{x}}$ (constructed from $\mathcal{S}$ by (4.6)) is positive.

(iii) For all $n \geqslant 1, \mathrm{x} \in \mathrm{T}^{n}, \lambda, \mu \in \mathrm{M}_{n}(\mathbb{C})_{++}, t \geqslant 0$ and $A \in \mathrm{M}_{n}(\mathrm{~V})$,

$$
\left\|\left(\lambda \cdot \varpi_{t}^{\mathbf{x}}\right)^{-1 / 2} \mathcal{P}_{t}^{\mathbf{x}}\left(\lambda^{1 / 2} A \mu^{1 / 2}\right)\left(\mu \cdot \varpi_{t}^{\mathbf{x}}\right)^{-1 / 2}\right\| \leqslant\|A\| .
$$

Proof. Suppose that $\mathrm{V}$ is an operator space in $B\left(\mathfrak{h} ; \mathfrak{h}^{\prime}\right)$.

(i $\Rightarrow$ ii): Let $k$ be a CC cocycle on $\mathrm{V}$. Then, by Corollary 1.2 , the cocycle $\widetilde{k}$ on $\widetilde{\mathrm{V}}$ is $\mathrm{CP}$ and unital, so by Proposition 5.2 each semigroup $\widetilde{\mathcal{P}}^{\mathbf{x}}$ is positive.

(ii $\Leftrightarrow$ iii): Fix $n \in \mathbb{N}, \mathbf{x} \in \mathrm{T}^{n}$ and $t \geqslant 0$, and let $\widetilde{\mathcal{P}}^{\mathbf{x}}$ be the semigroup constructed from $\mathcal{P}^{\mathrm{x}}$ as in (4.5). Under the identification of $\mathrm{M}_{n}(\widetilde{\mathrm{V}})$ given in (1.6), set

$$
M:=\left\{\left[\begin{array}{cc}
I_{\mathfrak{h}^{\prime}} \otimes \lambda & A \\
A^{*} & I_{\mathfrak{h}} \otimes \mu
\end{array}\right] \in \mathrm{M}_{n}(\widetilde{\mathrm{V}}): \lambda \text { and } \mu \text { are invertible }\right\} .
$$

By the characterisation (1.3) of nonnegative block matrices

$$
\begin{aligned}
& {\left[\begin{array}{cc}
I_{\mathfrak{h}^{\prime}} \otimes \lambda & A \\
A^{*} & I_{\mathfrak{h}} \otimes \mu
\end{array}\right] \in M_{+}} \\
& \quad \Longleftrightarrow \lambda, \mu \in \mathrm{M}_{n}(\mathbb{C})_{++} \text {and } A \in \mathrm{M}_{n}(\mathrm{~V}) \text { satisfies }\left\|\lambda^{-1 / 2} A \mu^{-1 / 2}\right\| \leqslant 1 .
\end{aligned}
$$

Since $M_{+}$is dense in the closed subset $\mathrm{M}_{n}(\widetilde{\mathrm{V}})_{+}$of $\mathrm{M}_{n}(\widetilde{\mathrm{V}})$, it follows that $\widetilde{\mathcal{P}}_{t}^{\mathbf{x}}$ is positive if and only if $\mathcal{P}_{t}^{\mathbf{x}}$ satisfies (5.9).

(iii $\Rightarrow$ i): Assume that (ii) and (iii) hold. Consider the family of semigroups $\widetilde{\mathcal{S}}:=\left\{\widetilde{\mathcal{P}}^{x, y}: x, y \in \mathrm{T}\right\}$ on the operator system $\widetilde{\mathrm{V}}$ constructed from the family of semigroups $\mathcal{S}$ by the prescription (4.5). Since each semigroup $\widetilde{\mathcal{P}}^{\mathbf{x}}$ is positive and satisfies $\widetilde{\mathcal{P}}_{t}^{\mathbf{x}}\left(I_{\mathfrak{h}^{\prime} \oplus \mathfrak{h}} \otimes \square_{n}\right)=I_{\mathfrak{h}^{\prime} \oplus \mathfrak{h}} \otimes \varpi_{t}^{\mathbf{x}}$, Theorem 5.4 ensures the existence of a $\mathrm{CP}$ unital cocycle $j$ on $\widetilde{V}$ whose associated semigroups include $\widetilde{\mathcal{S}}$. Letting $\varepsilon$ be the embedding $\mathrm{V} \rightarrow \widetilde{\mathrm{V}}, a \mapsto\left[\begin{array}{ll}0 & a \\ 0 & 0\end{array}\right]$ and $\pi$ be its left-inverse $\widetilde{\mathrm{V}} \rightarrow \mathrm{V},\left[\begin{array}{cc}\lambda & a \\ b & \mu\end{array}\right] \mapsto a$, the prescription $k_{t}:=\left(\pi \otimes_{\mathrm{M}} \operatorname{id}_{B(\mathcal{F})}\right) \circ j_{t} \circ \varepsilon$ $(t \geqslant 0)$, defines a completely contractive QS cocycle on $\mathrm{V}$ whose associated semigroups include the family

$$
\left\{\left(\pi \circ \widetilde{\mathcal{P}}_{t}^{x, y} \circ \varepsilon\right)_{t \geqslant 0}: x, y \in \mathrm{T}\right\}
$$


which equals $\mathcal{S}$. Thus (i) holds.

Remark. The applicability of criterion (iii) derives from its manifest stability under pointwise limits.

Contraction operator quantum stochastic cocycles. In this section we give our final characterisation. It is derived from the characterisation of CC cocycles in part (iii) of Theorem 5.10, via the correspondence between $\mathrm{CB}$ cocycles on $|\mathfrak{h}\rangle$ and bounded operator cocycles on $\mathfrak{h}$ given in Proposition 4.5 .

Theorem 5.11. Let $\mathrm{T}$ be a total subset of $\mathrm{k}$ containing 0 and let $S=\left\{P^{x, y}\right.$ : $x, y \in \mathrm{T}\}$ be a family of semigroups on a Hilbert space $\mathfrak{h}$. Then there is a left contraction operator cocycle on $\mathfrak{h}$ whose associated semigroups include $S$ if and only if for all $n \geqslant 1, \lambda, \mu \in \mathrm{M}_{n}(\mathbb{C})_{++}, \mathrm{x} \in \mathrm{T}^{n}, t \geqslant 0$ and $A \in \mathrm{M}_{n}(|\mathfrak{h}\rangle)$,

$$
\left\|\left(\lambda \cdot \varpi_{t}^{\mathbf{x}}\right)^{-1 / 2}\left(P_{t}^{\mathbf{x}} \cdot\left(\lambda^{1 / 2} A \mu^{1 / 2}\right)\right)\left(\mu \cdot \varpi_{t}^{\mathbf{x}}\right)^{-1 / 2}\right\| \leqslant\|A\| .
$$

Proof. This now follows from Proposition 4.5, the identities (4.10) and Theorem 5.10.

This theorem has led to new results on QS differential equations with unbounded coefficients for contraction operator processes $\left(\left[\mathrm{LW}_{4}\right]\right)$. Moreover, a very recent infinitesimal analysis of holomorphic contraction operator QS cocycles ([LiS]), which goes beyond the realm of quantum stochastic differential equations as currently understood, is also underpinned by this characterisation.

\section{REFERENCES}

[AFR] L. Accardi, F. Fagnola and M. Röckner, Weak, strong, and four semigroup solutions of classical stochastic differential equations: an example, in, "Stochastic Partial Differential Equations and Applications VII," Lecture Notes in Pure 8 Applied Mathematics 245, Chapman \& Hall/CRC, Boca Raton, 2006, pp. 1-6.

[AFL] L. Accardi, A. Frigerio and J.T. Lewis, Quantum stochastic processes, Publ. Res. Inst. Math. Sci. 18 (1982), no. 1, 97-133.

$\left[\mathrm{AK}_{1}\right] \quad$ L. Accardi and S.V. Kozyrev, The stochastic limit of quantum spin systems, in, "Proceedings, 3rd Tohwa International Conference on Statistical Physics," eds. M. Tokuyama and H.E. Stanley, AIP Proceedings 519, Springer-Verlag, Berlin, 2000 .

[AK $\mathrm{AK}_{2} \quad-\quad$, On the structure of Markov flows, Chaos Solitons Fractals 12 (2001) no. 14-15, 2639-2655.

$\left[\mathrm{AK}_{3}\right] \quad-$ - Lectures on quantum interacting particle systems, in "Quantum Interacting Particle Systems" QP-PQ 14 World Scientific, 2002, pp. 1-195.

[AcM] L. Accardi and A. Mohari, On the structure of classical and quantum flows, $J$. Funct. Anal. 135 (1996) no. 2, 421-455.

[Ar 1 W. Arveson, The domain algebra of a CP-semigroup, Pacific J. Math. 203 (2002), no. 1, 67-77, arXiv:math.OA/0005251.

[Ar $\mathrm{Ar}_{2} \quad$ - - "Noncommutative Dynamics and E-semigroups," Springer Monographs in Mathematics, Springer-Verlag, New York, 2003.

[BBLS] S.D. Barreto, B.V.R. Bhat, V. Liebscher and M. Skeide, Type I product systems of Hilbert modules, J. Funct. Anal. 212 (2004), 121-181.

$[\mathrm{BeW}]$ A.C.R. Belton and S.J. Wills, Quantum stochastic dilations on a UHF algebra, in preparation.

[Bha] B.V. Rajarama Bhat, Cocycles of CCR flows, Mem. Amer. Math. Soc. 149 no. 709 (2001). 
[BHS] L. Bouten, R. van Handel and A. Silberfarb, Approximation and limit theorems for quantum stochastic models with unbounded coefficients, J. Funct. Anal. 254 (2008), 3123-3147.

[BLM] D.P. Blecher and C. Le Merdy, "Operator Algebras and their Modules - an Operator Space Approach," Oxford University Press, Oxford, 2004.

[Bra] W.S. Bradshaw, Stochastic cocycles as a characterisation of quantum flows, Bull. Sci. Math. (2) 116 (1992) no. 1, 1-34.

$[\mathrm{BrR}] \quad$ O. Bratelli and D.W. Robinson, "Operator Algebras and Quantum Statistical Mechanics II: Equilibrium States, Models in Quantum Statistical Mechanics," Corrected 2nd Edition, Springer-Verlag, Heidelberg, 2002.

[Dav] E.B. Davies, "One-parameter Semigroups," Academic Press, London, 1980.

[EfR] E.G. Effros and Z.-J. Ruan, "Operator Spaces," Oxford University Press, Oxford, 2000 .

[Fag] F. Fagnola, A simple singular quantum Markov semigroup, in, "Stochastic Analysis and Mathematical Physics (Santiago, 1998)," Trends in Mathematics, Birkhäuser, Boston, 2000, pp. 73-87.

[FaS] F. Fagnola and K.B. Sinha, Quantum flows with unbounded structure maps and finite degrees of freedom, J. London. Math. Soc. 48 (1993) no. 3, 537-551.

[FoF] C. Foias and A.E. Frazho, The commutant lifting approach to interpolation problems, in, "Operator Theory: Advances and Applications," 44, Birkhäuser Verlag, Basel, 1990.

[GLSW] D. Goswami, J.M. Lindsay, K.B. Sinha and S.J. Wills, Dilation of Markovian cocycles on a von Neumann algebra, Pacific J. Math. 211 (2003) no. 2, 221-247.

[GKS] V. Gorini, A. Kossakowski and E.C.G. Sudarshan, Completely positive dynamical semigroups of $N$-level systems, J. Math. Phys. 17 (1976) no. 5, 821-825.

[Jou] J.-L. Journé, Structure des cocycles markoviens sur l'espace de Fock, Probab. Theory. Related Fields $\mathbf{7 5}$ (1987) no. 2, 291-316.

[KaR] R.V. Kadison and J.R. Ringrose, "Fundamentals of the theory of operator algebras, Vol. II: Advanced Theory," (Corrected reprint), Graduate Studies in Mathematics 16, American Mathematical Society, Providence, 1997.

[Lie] V. Liebscher, How to generate Markovian cocycles on Boson Fock space, Infin. Dimens. Anal. Quantum Probab. Relat. Top. 4 (2001) no. 2, 215-219.

[L] J.M. Lindsay, Quantum stochastic analysis - an introduction, in, "Quantum Independent Increment Processes," eds. U. Franz ES M. Schürmann, Lecture Notes in Mathematics 1865, Springer, Heidelberg, 2005.

[LiP] J.M. Lindsay and K.R. Parthasarathy, On the generators of quantum stochastic flows, J. Funct. Anal. 158 (1998) no. 2, 521-549.

[LiS] J.M. Lindsay and K.B. Sinha, Holomorphic quantum stochastic contraction cocycles, in preparation.

[LiT] J.M. Lindsay and O. Tripak, Matrix space tensor products as mapping spaces, in preparation.

$\left[\mathrm{LW}_{1}\right] \quad$ J.M. Lindsay and S.J. Wills, Existence, positivity, and contractivity for quantum stochastic flows with infinite dimensional noise, Probab. Theory Related Fields 116 (2000) no. 4, 505-543.

$\left[\mathrm{LW}_{2}\right] \quad$ - - Markovian cocycles on operator algebras, adapted to a Fock filtration, $J$. Funct. Anal. 178 (2000) no. 2, 269-305.

$\left[\mathrm{LW}_{3}\right] \quad-$ - Existence of Feller cocycles on a $C^{*}$-algebra, Bull. London Math. Soc. 33 (2001) no. 5, 613-621.

$\left[\mathrm{LW}_{4}\right] \quad-$ - Quantum stochastic operator cocycles via associated semigroups, Math. Proc. Cambridge Philos. Soc. 142 (2007) no. 3, 535-556; Construction of some quantum stochastic operator cocycles by the semigroup method, Proc. Indian Acad. Sci. Math. Sci. 116 (2006) no. 4, 519-529.

$\left[\mathrm{LW}_{5}\right] \quad \ldots$, Quantum stochastic cocycles and completely bounded semigroups on operator spaces II, Preprint, 2010.

[Mey] P.-A. Meyer, "Quantum Probability for Probabilists," 2nd Edition, Lecture Notes in Mathematics 1538 Springer, Heidelberg, 1993. 
[Neu] M. Neufang, Amplification of completely bounded operators and Tomiyama's slice maps, J. Funct. Anal. 207 (2004) no. 2, 300-329.

[Par] K.R. Parthasarathy, "An Introduction to Quantum Stochastic Calculus," Birkhäuser, Basel, 1992.

[Pau V.I. Paulsen, "Completely Bounded Maps and Operator Algebras," Cambridge University Press, Cambridge, 2002.

[Pin] M.A. Pinsky, Stochastic integral representation of multiplicative operator functionals of a Wiener process, Trans. Amer. Math. Soc. 167 (1972), 89-104.

[Pis] G. Pisier, "Introduction to Operator Space Theory," London Mathematical Society Lecture Note Series, 294 Cambridge University Press, Cambridge, 2003.

[Sch] W.J. Schreiner, Matrix regular operator spaces, J. Funct. Anal. 152 (1998) no. $1,136-175$.

[SiS] A.M. Sinclair and R.R. Smith, "Hochschild Cohomology of von Neumann Algebras," London Mathematical Society Lecture Note Series, 203, Cambridge University Press, Cambridge, 1995.

[SiG] K.B. Sinha and D. Goswami, "Quantum Stochastic Processes and Noncommutative Geometry," Cambridge Tracts in Mathematics 169, Cambridge University Press, 2007.

[Sko] A.V. Skorohod, "Asymptotic Methods in the Theory of Stochastic Differential Equations," Translations of Mathematical Monographs 78, American Mathematical Society, Providence, 1989.

[Tom] J. Tomiyama, Tensor products and approximation problems of $C^{*}$-algebras, Publ. RIMS, Kyoto Univ. 11 (1975), 163-183.

[W] S.J. Wills, On the generators of quantum stochastic operator cocycles, Markov Proc. Related Fields 13 (2007) no. 1, 191-211.

Department of Mathematics and Statistics, Lancaster University, LanCASTER LA1 4YF, UK

E-mail address: j.m.lindsay@lancs.ac.uk

School of Mathematical Sciences, University College Cork, Cork, Ireland E-mail address: s.wills@ucc.ie 